\title{
Mechanisms of Mixed Th1/Th2 Responses in Mice Induced by Albizia julibrissin Saponin Active Fraction by in Silico Analysis
}

\author{
Jing Du ${ }^{1}$, Junjie Jin ${ }^{1,2}$, Juanjuan Wang ${ }^{1}$ and Hongxiang Sun ${ }^{1, * \mathbb{C}}$ \\ 1 Key Laboratory of Animal Virology of Ministry of Agriculture, College of Animal Sciences, Zhejiang \\ University, Hangzhou, Zhejiang 310058, China; 11317036@zju.edu.cn (J.D.); 2013038@wzvcst.edu.cn (J.J.); \\ jjwang@asieris.cn (J.W.) \\ 2 College of Animal Sciences, Wenzhou Vocational College of Science and Technology, Wenzhou 325006, China \\ * Correspondence: sunhx@zju.edu.cn; Tel./Fax: +86-570-8898-2091
}

Received: 24 December 2019; Accepted: 23 January 2020; Published: 27 January 2020

check for updates

\begin{abstract}
The purified active fraction of Albizia julibrissin saponin (AJSAF) is an ideal adjuvant candidate that improves antigen-specific both cellular and humoral immune responses and elicits mixed Th1/Th2 responses, but its mechanisms remain unclear. The key features of action of AJSAF were investigated in mice immunized with Newcastle disease virus-based recombinant influenza vaccine (rL-H5) and AJSAF at the same leg (AJSAF+rL-H5) or different legs (AJSAF/rL-H5). The adjuvant activity of AJSAF on rL-H5 is strictly dependent on their spatial colocalization. Serum $\mathrm{H} 5$ antigen (H5Ag)-specific IgG, IgG1, IgG2a, and IgG2b antibody titers in AJSAF+rL-H5 group were significantly higher than those in AJSAF/rL-H5 group. The mechanisms of selectivity of Th1 or Th2 in mice induced by AJSAF was explored by the transcriptomic and proteomic profiles of H5Ag-stimulated splenocytes from the immunized mice using gene microarray and two-dimensional difference gel electrophoresis coupled with matrix-assisted laser desorption/ionization time-of-flight mass spectrometry. Compared to rL-H5 alone, AJSAF/rL-H5 induced more differentially expressed genes (DEGs) than AJSAF+rL-H5, whereas AJSAF+rL-H5 upregulated higher mRNA expression of Th1 (T-bet, IFN- $\gamma$, TNF- $\alpha$, IL-12 $\beta$, and IL-12R $\beta 1$ ) and Th2 (IL-10 and AICDA) immune response genes. The neutrophil response and its derived S100A8 and S100A9 might be involved in the AJSAF-mediated Th1 response. Meanwhile, AJSAF might induce the adaptive immune responses by improving a local innate immune microenvironment. These findings expanded the current knowledge on the mechanisms of action of saponin-based adjuvants, and provided new insights into how adjuvants shape adaptive immune responses.
\end{abstract}

Keywords: Albizia julibrissin saponin; adjuvant; Newcastle disease virus-based recombinant influenza vaccine; adaptive immunity; transcriptome and proteome; bioinformatics

\section{Introduction}

Adjuvants are essential components of new generation vaccines. Adjuvants not only augment the adaptive immune response to vaccines, but also induce the most effective immune response types for specific pathogens. Th1 or Th2 responses generated upon antigenic stimulation can be modulated in vivo depending on the adjuvant used for immunization [1]. The Th1 immunity, correlated with the cellular immune response, is required for therapeutic cancer vaccines, as well as vaccines directed against intracellular pathogens such as viruses, certain bacteria, and parasite [2]. The Th2 immunity, which controls the humoral immune response, is effective for protection against extracellular pathogens including most bacteria and certain viruses [3]. The Th1/Th2 paradigm provides a useful model for understanding the mechanisms of adjuvant and the basis for the rational design of new adjuvants. 
How the nature of adjuvants determines T-cell response type is an area of great interest, and the mechanisms responsible for this regulation are only presently being unraveled. The adjuvants are usually classified into pattern recognition receptor (PRR)-dependent and -independent types. An increasing number of studies have focused on pathogen-associated molecular patterns (PAMPs) as candidate Th1 adjuvants, which were recognized by PRRs especially toll-like receptors (TLRs) to activate dendritic cells (DCs) resulting in the generation of IL-12p70 or interferons (IFNs) critical for the Th1 polarization [4]. 3-O-desacyl-4'-monophosphoryl lipid A (MPL), a TLR4 ligand, efficiently induced DC maturation and further enhanced Th1 responses through IL-12p70 [5]. PRR-independent adjuvants such as Alum and MF59 were empirically used and have been proved to be effective adjuvants, whereas incomplete understanding of their mechanisms has seriously hampered further development. The most widely used adjuvant Alum was reported to exert adjuvant activity through inducing NLRP3 inflammasome [6-8] and damage-associated molecular patterns (DAMPs) such as uric acid (UA) [9] and host DNA $[10,11]$. However, some studies showed their limited roles in Alum adjuvant activity or even remained contradicted with other reports [12,13]. Therefore, although there have been some reports on the mechanism of Th1 or Th2 selectivity, the details concerning integrated mechanisms of action of adjuvants remain unclear.

The purified active fraction of saponin from the stem bark of Albizia julibrissin Durazz. (AJSAF) would be a promising adjuvant candidate for vaccines. It has been proved to improve antigen-specific cellular and humoral immune responses, and simultaneously elicit mixed Th1/Th2 responses in mice to the H5 avian influenza vaccine [14] and porcine reproductive and respiratory syndrome virus vaccine [15]. In our previous studies, it was found that the colocalization of AJSAF with antigen or not significantly affected its adjuvant activity in mice. In fact, the adjuvant activities of other adjuvants such as AS03, chitosan, and phytol derivatives were also reported to depend on their spatial and temporal colocalization with the antigen [16].

In this study, the effects of the colocalization of AJSAF with antigen or not on its adjuvant activity were investigated in mice using the Newcastle disease virus-based recombinant influenza vaccine (rL-H5). Further, the mechanisms resulting in the differences of antigen-specific immune responses between two injection regimens were explored using gene microarray and two-dimensional difference gel electrophoresis coupled with matrix-assisted laser desorption/ionization time-of-flight mass spectrometry (2D DIGE-MALDI-TOF-MS).

\section{Materials and Methods}

\subsection{Materials}

Newcastle disease virus (NDV)-based recombinant influenza vaccine (rL-H5) and H5 subtype AIV hemagglutination inhibition detecting antigen $(\mathrm{H} 5 \mathrm{Ag})$ were purchased from the Harbin Weike Biotechnology Development Co., Heilongjiang, China. RPMI medium was from Hyclone/GE Healthcare, Logan, UT, USA; fetal bovine serum (FBS) was from Gibco, Grand Island, NY, USA. Rabbit anti-mouse IgG peroxidase conjugate were purchased from Sigma Chemical Co., St. Louis, MO, USA; goat anti-mouse IgG1 and IgG2b peroxidase conjugates were from Southern Biotech. Assoc., Birmingham, AL, USA; goat anti-mouse IgG2a peroxidase conjugates were from Abcam, Cambridge, UK. Trizol reagent was purchased from Invitrogen, Carlsbad, CA, USA; revert Aid ${ }^{\mathrm{TM}} \mathrm{M}-\mathrm{MuLV}$ reverse transcriptase was from Fermentas, USA; diethylpyrocarbonate (DEPC), ribonuclease inhibitor, and oligo(dT) 18 were from Shanghai Sangon Biological Engineering Technology Co., Ltd., Shanghai, China; FastStart Universal SYBR Green Master (ROX) was from Roche Diagnostics Ltd., Shanghai, China. Agilent $4 \times 44 \mathrm{k}$ whole mouse genome microarray was provided from Agilent Technologies. Santa Clara, CA, USA.

\subsection{Preparation and Characterization of AJSAF}

AJSAF was prepared and characterized as previously described [15]. A total of 29 saponins including 10 new compounds in AJASF were identified and characterized by a high-performance liquid 
chromatography coupled with quadrupole time-of-flight mass spectrometry based on accurate mass database [17]. The endotoxin level in an AJSAF solution of $2 \mathrm{mg} / \mathrm{mL}$ was measured to be $0.253 \pm 0.004$ endotoxin units/ml by a tachypleus amebocyte lysate assay, indicating that AJSAF used in this study could be excluded from endotoxin contamination.

\subsection{Mice}

Female BALB/c mice aged 4-6 weeks were purchased from the Shanghai Experimental Animal Center, Chinese Academy of Sciences, Shanghai, China. Mice were acclimatized for one week prior to use. Rodent laboratory chow and tap water were provided ad libitum and maintained under controlled conditions with a temperature of $24 \pm 1{ }^{\circ} \mathrm{C}$ and humidity of $50 \% \pm 10 \%$, and a 12/12 h light/dark cycle. All experiments were in compliance with the People's Republic of China legislation on the use and care of laboratory animals, and followed the guidelines established by the Institute of Laboratory Animals of Zhejiang University, and approved by the University Animal Experimental Committee (no. 14878).

\subsection{Immunization}

To evaluate the adjuvant effects of AJSAF on rL-H5 vaccine, mice were divided into four groups, each consisting of five or 12 mice. Animals were immunized subcutaneously (s.c.) with rL-H5 $\left(10^{6}\right.$ EID $_{50} /$ dose $)$ [18] alone or in combination with AJSAF (100 $\left.\mu \mathrm{g}\right)$ at the same leg (AJSAF+rL-H5) or at different legs (AJSAF/rL-H5) on Day 1. A boosting injection was given two weeks later. Animals injected with $200 \mu \mathrm{L}$ of PBS were included as a negative control. Sera and splenocytes were collected two weeks after the second immunization for measurement of the H5Ag-specifc antibody, gene microarray, and quantitative real-time PCR (qRT-PCR).

For 2D DIGE-MALDI-TOF-MS, mice were divided into two groups, each consisting of 12 mice. Animals were immunized s.c. with rL-H5 alone or in combination with AJSAF $(100 \mu \mathrm{g})$ at the same leg (AJSAF+rL-H5) on Day 1. A boosting injection was given two weeks later. Splenocytes were collected two weeks after the second immunization.

\subsection{Measurement of H5Ag-Specific Antibody}

The serum H5Ag-specific IgG, IgG1, IgG2a, and IgG2b antibodies were detected in individual serum samples by an indirect ELISA as previously described [18]. The optical density was measured in a BIO-RAD 680 ELISA reader at $492 \mathrm{~nm}$, where sets of sera samples have been subjected within and between group comparisons, and ELISA assays were performed on the same day for all of the samples.

\subsection{Quantitative Real-Time PCR (qRT-PCR)}

Splenocytes from immunized mice were incubated with H5Ag (final concentration 0.125 hemagglutinating units $(\mathrm{HAU}) / \mathrm{ml})$ for $12 \mathrm{~h}$. The total RNA was isolated with TRIzol reagent and reverse transcription was performed as previously [19]. The PCR was performed with FastStart universal SYBR Green Master (ROX) on a BioRad CFX96 system. Primers for qRT-PCR were synthesized by Shanghai Sangon Biological Engineering Technology Co., Ltd., China, and the sequences were listed in Supplementary Table S1. The qPCR cycling was performed as follows: Initial denaturation at $95^{\circ} \mathrm{C}$ for $10 \mathrm{~min}$ followed by 40 cycles of denaturation at $95^{\circ} \mathrm{C}$ for $10 \mathrm{~s}$, and annealing for $60 \mathrm{~s}$. GAPDH was used as an endogenous control. The mRNA expression levels of the tested genes relative to GAPDH were determined using the $2^{-\Delta \Delta C t}$ method and as fold induction [19].

\subsection{Microarray Analysis}

The splenocytes were stimulated with H5Ag (final concentration $0.125 \mathrm{HAU} / \mathrm{ml}$ ) for $12 \mathrm{~h}$. Splenocytes from four mice per group were pooled and total RNA was extracted using a TRIzol reagent and further purified with the RNeasy®Mini kit (Qiagen). RNA quality was assessed by the 2100 Bioanalyzer automated microfluidic system in combination with the RNA 6000 Nano kit (Agilent) 
following the producer's protocol. Agilent $4 \times 44 \mathrm{k}$ whole mouse genome microarray representing 41,174 probes was used, and the RNA labelling and microarray hybridization were carried out according to the Agilent one-color microarray-based gene expression analysis protocol. Hybridized microarrays were scanned with a DNA microarray scanner (G2565BA, Agilent Technologies) and features were extracted using the Feature Extraction software 10.7 (Agilent Technologies) using default protocols and settings (scan resolution $=5 \mu \mathrm{m}$, PMT 100\%). Data pre-processing and differential expression analysis of the gene expression data were done in $\mathrm{R}$ (v2.10.0, Bioconductor). Data were normalized between arrays using the quantile method (GENESPRING12.0). Normalized expression data was subjected to $\log 2$ transformation. Significantly regulated probes were selected by the following cutoff: Fold change (FC) $>2$ and $p<0.05$. Gene set enrichment analysis (GSEA) on "adaptive immune response (GO: 0002250)" was performed using the analysis software provided by the Broad Institute (Cambridge, MA) [20]. Venn diagram was performed in $\mathrm{R}$ with Vennerable package (v3.5.1). The common genes of AJSAF+rL-H5 and AJSAF/rL-H5 were defined as differentially expressed genes (DEGs) in both groups but with no significantly different expression; AJSAF+rL-H5-specific genes were defined as the DEGs in AJSAF+rL-H5 group with significantly different expression compared to AJSAF/rL-H5, while AJSAF/rL-H5-specific genes were defined as DEGs in AJSAF/rL-H5 group with significantly different expression compared to AJSAF+rL-H5. The pathway and function enrichment analysis of each gene set was performed using Metascape (http://metascape.org/gp/index.html\#/main/step1) [21]. The network analysis of protein-protein interactions (PPI) within each gene set was carried out based on IMEx Interactome by InnateDB (http://www.innatedb.com/) [22] and visualized using Cytoscape (v3.6.0) [23].

\subsection{Two-Dimensional Difference Gel Electrophoresis (2D DIGE)}

The splenocytes were stimulated with $\mathrm{H} 5 \mathrm{Ag}$ (final concentration $0.125 \mathrm{HAU} / \mathrm{ml}$ ) for $24 \mathrm{~h}$. Splenocytes from four mice per group were pooled and solubilized in the DIGE lysis buffer by ultrasound on ice ( $80 \mathrm{~W}, 10 \mathrm{~s}$ each time, 5 times, $15 \mathrm{~s}$ apart). The supernatant was collected by centrifugation at $12,000 \times \mathrm{g}$ for $45 \mathrm{~min}$ and the protein contents were determined using the Protein Assay kit (Bio-Rad). For two-dimensional gel electrophoresis (2-DE), $100 \mu \mathrm{g}$ and $1 \mathrm{mg}$ of proteins were loaded onto analytical and preparative gels, respectively. The Ettan IPGphor Isoelectric Focusing System (GE Amersham) and pH 3-10 immobilized pH gradient (IPG) strips (13 cm, nonlinear; GE Healthcare) were used for isoelectric focusing (IEF). The IPG strips were rehydrated for $12 \mathrm{~h}$ in $250 \mu \mathrm{L}$ of rehydration buffer containing the protein samples. IEF was performed in four steps: $30 \mathrm{~V}$ for $12 \mathrm{~h}$, $500 \mathrm{~V}$ for $1 \mathrm{~h}, 1000 \mathrm{~V}$ for $1 \mathrm{~h}$, and $8000 \mathrm{~V}$ for $8 \mathrm{~h}$. The gel strips were equilibrated for $15 \mathrm{~min}$ in an equilibration buffer ( $50 \mathrm{mM}$ Tris- $\mathrm{HCl}$ ( $\mathrm{pH} 8.8$ ), $6 \mathrm{M}$ urea, $2 \% \mathrm{SDS}, 30 \%$ glycerol, and $1 \% \mathrm{DTT}$ ). This step was repeated using the same buffer with $4 \%$ iodoacetamide in place of $1 \%$ DTT. The strips were then subjected to the second-dimensional electrophoresis after transfer onto $12.5 \%$ SDS-polyacrylamide gels. Electrophoresis was performed using the Hofer SE 600 system (GE Amersham) at $15 \mathrm{~mA}$ per gel for $30 \mathrm{~min}$, followed by $30 \mathrm{~mA}$ per gel until the bromophenol blue reached the end of the gel. Three replicates were performed for each sample. Protein spots in the nine gels were visualized by silver staining to estimate the 2-DE feasibility of all samples.

For 2D DIGE, $50 \mu \mathrm{g}$ proteins per sample was labeled with 400 pmol of Cy3 or Cy 5 fluorescent dyes (GE Healthcare) and $50 \mu \mathrm{g}$ of equal mixture from three samples was labeled with $400 \mathrm{pmol}$ of $\mathrm{Cy} 2$ fluorescent dye (GE Healthcare) as an internal standard. The labeling reaction was carried out in the dark on ice for $30 \mathrm{~min}$, and quenched with $10 \mathrm{mM}$ lysine for $10 \mathrm{~min}$. The Cy2, Cy3, and Cy5-labeled samples were mixed and 2-DE was performed as described above. The images were acquired on a UMax Powerlook 2110XL (GE Healthcare) at the excitation/emission of 488/520, 532/580, and 633/670 $\mathrm{nm}$, respectively, and analyzed with the DeCyder Image-Quant ${ }^{\mathrm{TM}}$ software (GE Healthcare). Protein spots were represented in all gels $(n=3)$ with expression level greater than 1.2-FC and $p<0.05$ were defined as being differentially expressed and selected for further characterization. 


\subsection{Protein Identification by MALDI-TOF-MS}

All the differentially expressed spots were selected and excised manually from the preparative gels. Protein spots of interest were cut from the preparative gels, destained for $20 \mathrm{~min}$ in $30 \mathrm{mM}$ potassium ferricyanide/100 $\mathrm{mM}$ sodium thiosulfate $(1: 1 \mathrm{v} / \mathrm{v})$, and washed with Milli-Q water until the gels were destained. The spots were incubated in $0.2 \mathrm{M} \mathrm{NH}_{4} \mathrm{HCO}_{3}$ for $20 \mathrm{~min}$ and then lyophilized. Each spot was digested overnight in $12.5 \mathrm{ng} / \mu \mathrm{L}$ trypsin in $25 \mathrm{mM} \mathrm{NH}_{4} \mathrm{HCO}_{3}$. The peptides were extracted three times with $60 \%$ acetonitrile $(\mathrm{ACN}) / 0.1 \%$ trifluoroacetic acid (TFA). The extracts were pooled and dried completely by a vacuum centrifuge.

MS and MS/MS data for protein identification were obtained by using a MALDI-TOF-TOF instrument (5800 proteomics analyzer; Applied Biosystems). Instrument parameters were set using the 4000 Series Explorer software (Applied Biosystems). The MS spectra were recorded in the reflector mode in a mass range from 800 to 4000 with a focus mass of 2000. The TOF/TOF calibration mixtures (AB SCIEX) were used to calibrate the spectrum to a mass tolerance within $10 \mathrm{ppm}$. The MS spectra were processed using the TOF-TOF Series Explorer software (v4.0, AB SCIEX). At least 1000 laser shots were typically accumulated with a laser pulse rate of $400 \mathrm{~Hz}$ in the MS mode, whereas in the MS/MS mode spectra up to 2000 laser shots were acquired and averaged with a pulse rate of $1000 \mathrm{~Hz}$. For MS calibration, autolysis peaks of trypsin $\left([\mathrm{M}+\mathrm{H}]^{+} 842.5100\right.$ and 2211.1046) were used as internal calibrates, and the most intense ion signals (up to 10) were selected as precursors for MS/MS acquisition, excluding the trypsin autolysis peaks and the matrix ion signals.

The peptide mass finger printing (PMF) and MS/MS queries were performed using the MASCOT search engine 2.2 (Matrix Science, London, UK) embedded into the GPS-Explorer Software 3.6 (Applied Biosystems) on the NCBI protein database with the following parameter settings: Mass accuracy $100 \mathrm{ppm}$, trypsin cleavage one missed cleavage allowed, carbamidomethylation set as fixed modification, oxidation of methionine was allowed as variable modification, and MS/MS fragment tolerance was set to $0.4 \mathrm{Da}$. A GPS Explorer protein confidence index $\geq 95 \%$ were used for further manual validation.

\subsection{Statistical Analysis}

The normality of the distribution of each variable was measured through means of the Kolmogorov-Smirnov test. Data were expressed as mean \pm SEM and examined for their statistical significance of difference with ANOVA and a Tukey post-hoc test. The calculations and graphs were produced using the Prism 7 software (GraphPad Software, San Diego, CA, USA). A $p$-value less than 0.05 were considered to be statistically significant.

\section{Results}

\subsection{Comparative Analysis of H5Ag-Specific Serum Antibody Response}

The serum H5Ag-specific IgG, IgG1, IgG2a, and IgG2b antibody levels were measured two weeks after the last immunization using ELISA, and the results were shown in Figure 1. The rL-H5 alone induced the low serum H5Ag-specific IgG and its isotypes antibody liters. The addition of AJSAF to rL-H5 (AJSAF+rL-H5) resulted in a significant increase in serum H5Ag-specific IgG, IgG1, $\operatorname{IgG} 2 \mathrm{a}$, and IgG2b antibody titers $(p<0.01$ or $p<0.001)$. However, the immunization by injection of AJSAF and rL-H5 in mouse different legs (AJSAF/rL-H5) only significantly enhanced the serum H5Ag-specific IgG and IgG1 titers in the rL-H5-immunized mice compared with the rL-H5 alone group $(p<0.05$ or $p<0.01$ ) (Figure 1A,B). There were, however, no significant differences in the serum H5Ag-specific IgG2a and IgG2b titers between rL-H5 alone and AJSAF/rL-H5 groups $(p>0.05)$ (Figure 1C,D). Moreover, the serum H5Ag-specific IgG, IgG1, IgG2a, and IgG2b antibody titers in AJSAF/rL-H5-immunized mice were significantly lower than those in the AJSAF+rL-H5 group $(p<0.05$, or $p<0.001)$. The antigen-specific IgG2a/2b and IgG1 are markers for Th1 and Th2 responses, 
respectively. Therefore, the adjuvant activity of AJSAF on Th1 and Th2 responses was dependent on the colocalization of AJSAF and antigen.

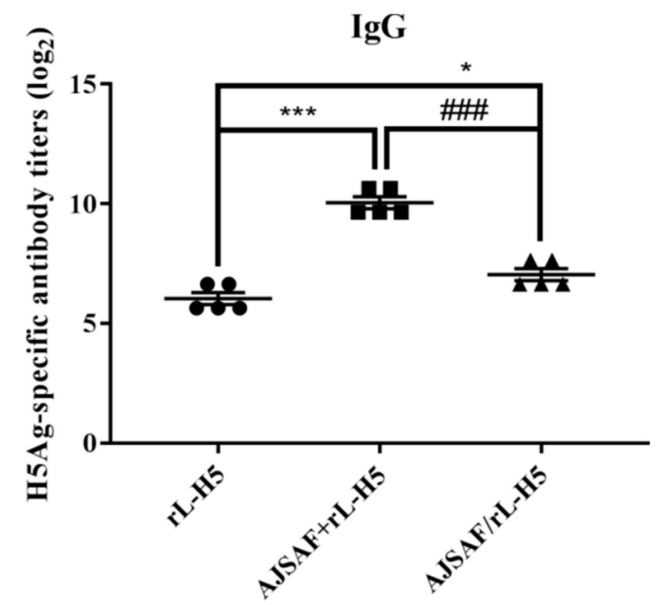

(A)

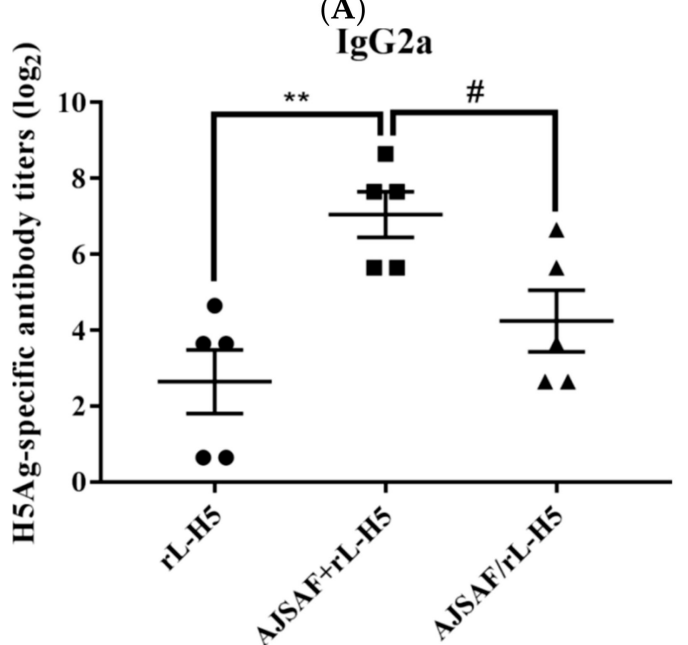

(C)

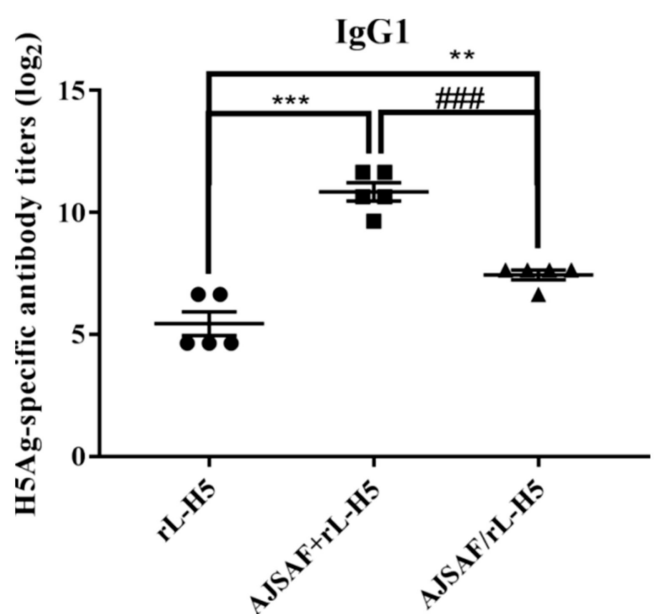

(B)

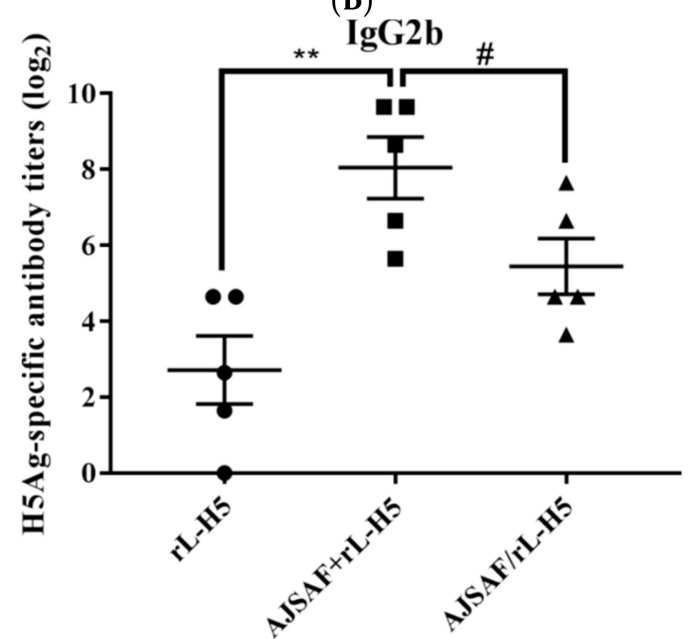

(D)

Figure 1. Serum H5Ag-specific IgG (A), IgG1 (B), IgG2a (C), and IgG2b (D) antibody titers in mice immunized with Newcastle disease virus-based recombinant influenza vaccine alone (rL-H5) or in combination with the purified active fraction of Albizia julibrissin saponin (AJSAF) at the same leg $(\mathrm{AJSAF}+\mathrm{rL}-\mathrm{H} 5)$ or different legs (AJSAF/rL-H5). The values are presented as mean $\pm \mathrm{SEM}(n=5)$. $P$-values were determined by ANOVA and a Tukey post-hoc test. Significant differences with the rL-H5 alone group were designated as ${ }^{*} p<0.05,{ }^{* *} p<0.01$, and ${ }^{* * *} p<0.001$; those with the AJSAF/rL-H5 group as ${ }^{\#} p<0.05,{ }^{\#} p<0.01$, and ${ }^{\# \#} p<0.001$.

\subsection{Comparative Analysis of Global Gene Expression}

To gain insight into the molecular mechanisms underlying the responses to the different regimens of AJSAF and rL-H5, gene microarray and 2D DIGE-MALDI-TOF-MS were used to analyze the transcriptomic and proteomic profiles of $\mathrm{H} 5 \mathrm{Ag}$-stimulated splenocytes from immunized mice (Figure 2). The splenocytes stimulated with $\mathrm{H} 5 \mathrm{Ag}$ for $12 \mathrm{~h}$ were subjected to microarray analysis. Six hundred and forty-two differentially expressed probes were identified in AJSAF+rL-H5 group relative to rL-H5 alone group with FC $>2$ and $p<0.05$ calculated on the three replicates (Figure 3A, right). Among them, 546 were upregulated and 96 were downregulated, corresponding to 411 and 66 genes, respectively, after correcting for redundant probes and excluding unknown genes. Unexpectedly, a larger number of DEGs were identified for AJSAF/rL-H5 group. There were 1504 differentially expressed probes in AJSAF/rL-H5 group compared to rL-H5 alone group. Among them, 1293 were upregulated and 
211 were downregulated, corresponding to 917 and 169 genes, respectively (Figure 3A, middle). The comparison of AJSAF+rL-H5 group with AJSAF/rL-H5 group revealed 330 differentially expressed probes, covering 178 upregulated and 152 downregulated probes, corresponding to 145 and 121 genes, respectively (Figure 3A, left). To confirm the validity of the microarray data, qRT-PCR was undertaken for putative six DEGs (S100A8, MCP-1/CCL2, IFN- $\gamma$, T-bet, FAM19A3, and IL-5), and the results were shown in Figure 3B and Figure S1. The qRT-PCR results were consistent with the microarray data except for IFN- $\gamma$. In qRT-PCR results, the mRNA expression levels of IFN- $\gamma$ in AJSAF/rL-H5 group were significantly lower than those in AJSAF+rL-H5 group $(p<0.001)$. There was, however, no significant difference between $\mathrm{rL}-\mathrm{H} 5$ alone and AJSAF/rL-H5 groups $(p<0.05)$. In microarray data, the mRNA expression levels of IFN- $\gamma$ in AJSAF/rL-H5 group were significantly lower than those in both rL-H5 alone and AJSAF+rL-H5 groups for two designed IFN- $\gamma$ probes $(p<0.001)$.

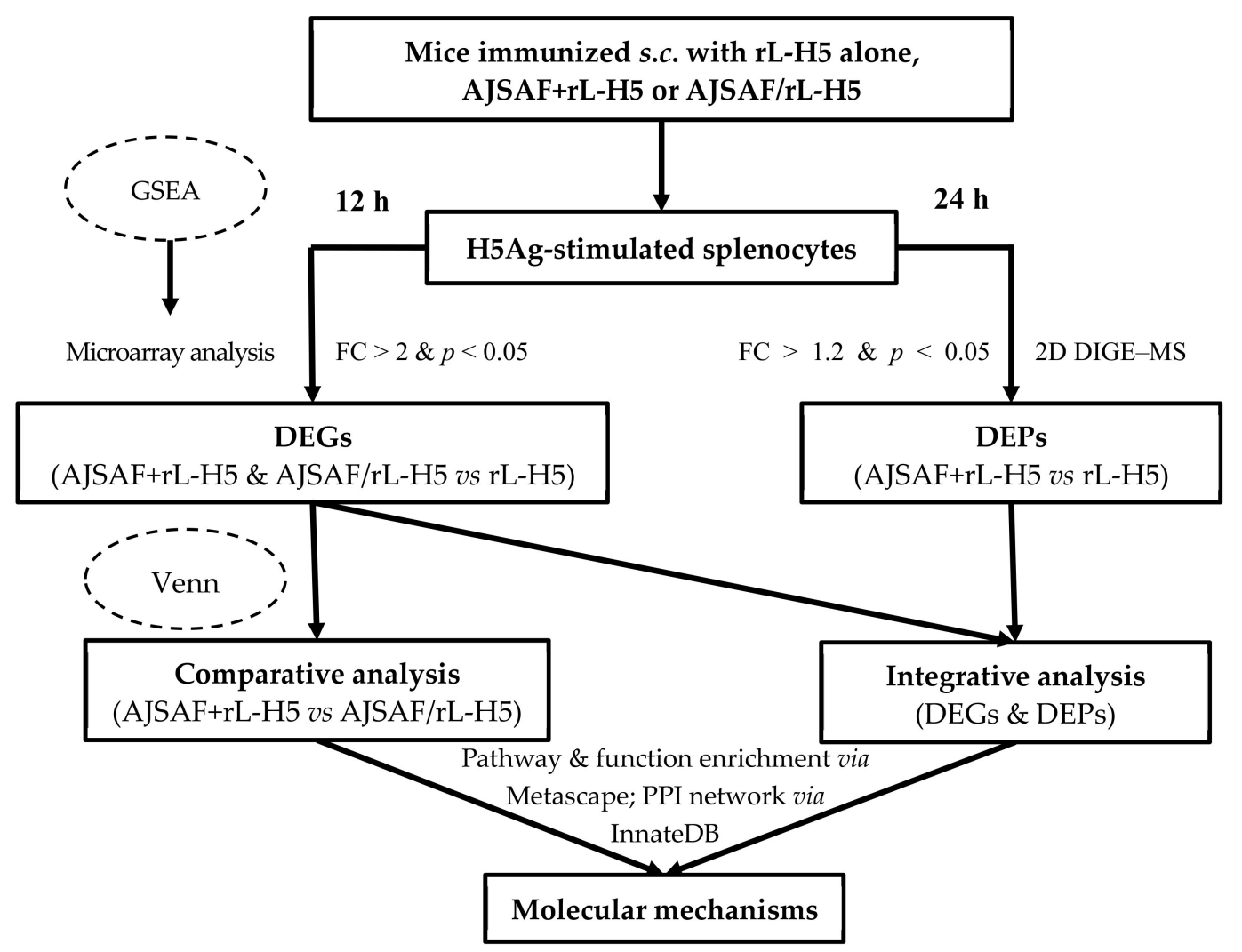

Figure 2. Workflow of the transcriptomic and proteomic analyses.

GSEA of DEGs in AJSAF+rL-H5 and AJSAF/rL-H5 groups compared to rL-H5 alone group was conducted to compare the statistically significant differences of gene expression in the defined gene sets across "adaptive immune response (GO: 0002250)". There was, however, no difference in the enriched gene sets in both AJSAF+rL-H5 and AJSAF/rL-H5 groups compared to rL-H5 alone group (FDR $>25 \%$, Figure S2). Therefore, an alternative was used to focus on enriched individual genes to identify functional molecular signatures (Figure 3C,D). Compared to rL-H5 alone group, CEACAM1 and CADM1 for "circulating antibody mediated immune response", as well as C3, C7, and C1RA/B for "T cell mediated cytotoxicity" were enriched in AJSAF+rL-H5 and AJSAF/rL-H5 groups. Compared to AJSAF/rL-H5 groups, the more upregulated genes involved in adaptive immunity, such as IFN- $\gamma$, T-bet (TBX21), ANXA1, IL-12RB1, TNF, and IL-12 $\beta$ for “Th1 immune response", IL-10, RSAD2, and IL-6 for "Th2 immune response", IL-17F for "Th17 immune response", and AICDA, XCL1, and IL-10 for "B cell mediated immunity", were enriched in AJSAF+rL-H5 group. Notably, T-bet was identified as a core enriched gene for Th1, Th2, and Th17 responses. T-bet is a core transcription factor promoting Th1 response [24] and negatively regulating Th2 and Th17 responses [25,26]. In addition, it was found 
that the heterogeneity of splenocytes could limit the sensitivity of gene expression such as IL-12 $\beta$, IL-6, and TNF $(1.5<\mathrm{FC}<2$ and $p<0.05)$.
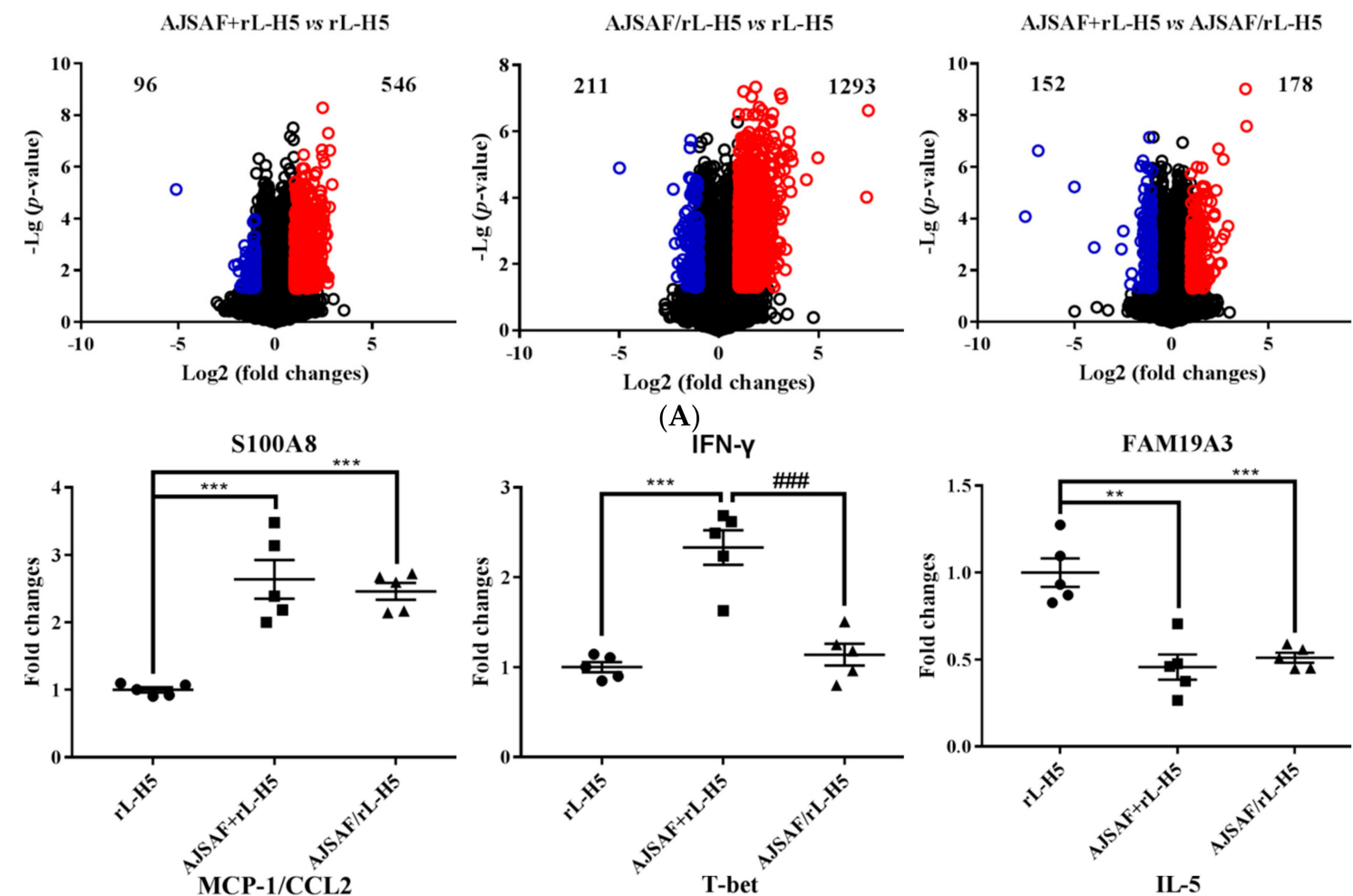

(A)
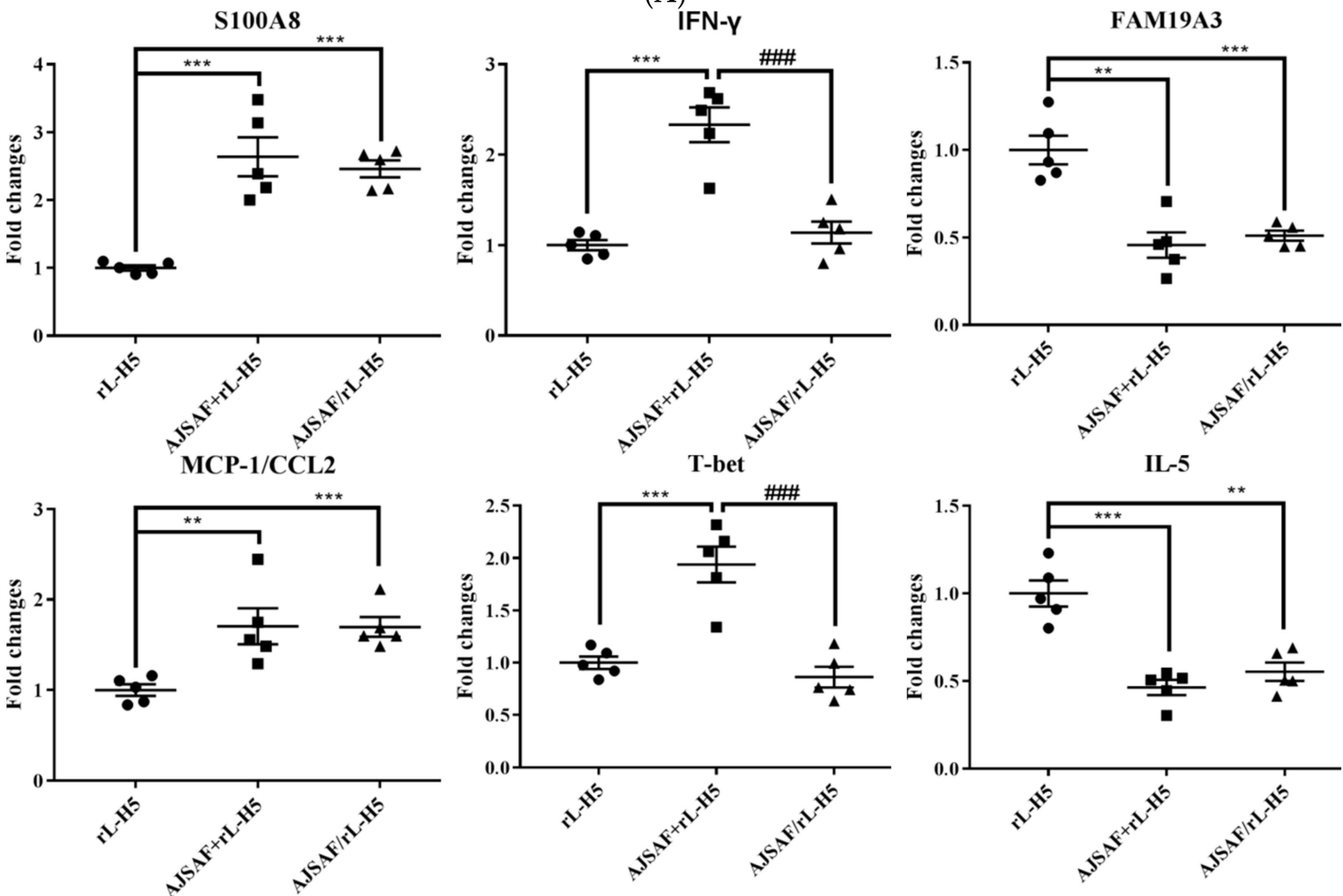

(B)

Figure 3. Cont. 

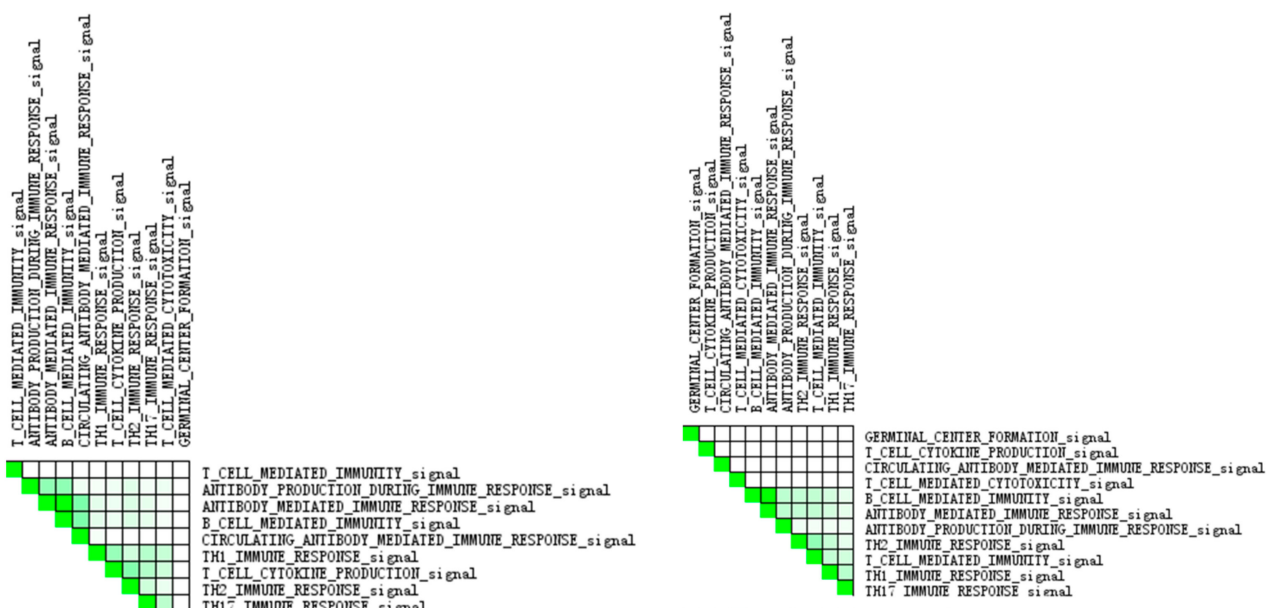

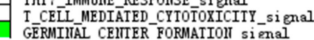
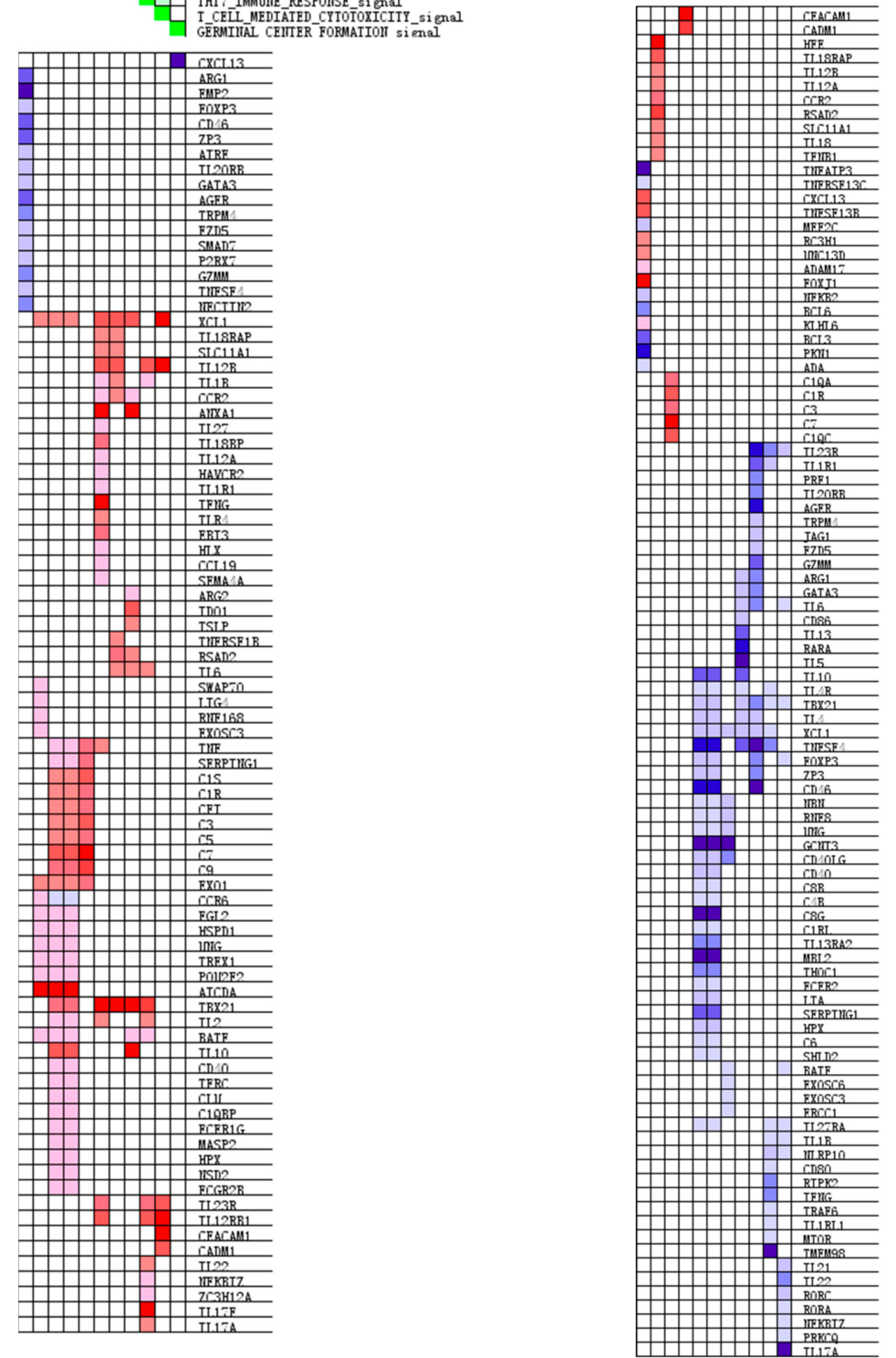

(C)

(D)

Figure 3. Expression profiles of differentially expressed genes (DEGs) in H5Ag-stimulated splenocytes from the mice immunized with rL-H5 alone (rL-H5) or in combination with AJSAF at the same leg (AJSAF+rL-H5) or different legs (AJSAF/rL-H5). (A) Volcano plots. (B) qRT-PCR verification. P-values 
were determined by ANOVA and a Tukey post-hoc test. The values are presented as mean \pm SEM $(n=5)$. Significant differences with rL-H5 alone group were designated as $*<0.05,{ }^{* *} p<0.01$, and ${ }^{* * *} p<0.001$; those with AJSAF/rL-H5 group as ${ }^{\#} p<0.05,{ }^{\# \#} p<0.01$, and ${ }^{\# \#} p<0.001$. (C,D) Heatmap of enriched DEGs on "adaptive immune response (GO: 0002250)" in AJSAF+rL-H5 (C) and AJSAF/rL-H5 (D) compared to rL-H5 alone using gene set enrichment analysis (GSEA).

\subsection{Pathway and Function Analysis of DEGs}

In view of that the colocalization of AJSAF with antigen was required for its adjuvant activity, to identify the gene signatures mediated its adjuvant activity, DEGs were classified into three categories: AJSAF+rL-H5-specific, AJSAF/rL-H5-specific, and their common genes, including 58, 138, and 323 DEGs, respectively (Figure 4A). Among AJSAF+rL-H5-specific DEGs, IFN- $\gamma$, IL-12R $\beta 1$, IL-10, T-bet, and AICDA were related to "adaptive immune response".

Next, the function enrichment analysis of DEGs was performed, and the results were shown in Figure 4B. The common DEGs were specifically enriched in "cell-cell adhesion", "myeloid leukocyte activation", and "neutrophil mediated immunity". These common DEGs were also involved in "Neutrophil degranulation" with a higher $p$-value $(p=1.438 \mathrm{E}-15)$. AJSAF+rL-H5-specific DEGs were enriched in "cell chemotaxis", "myeloid leukocyte migration", and "defense response to other organism" with a higher $p$-value than the other two categories. There were, however, no specific terms enriched in AJSAF/rL-H5-specific DEGs. The relationships of these enriched terms were visualized as an integrated network in Figure 4C. It revealed "myeloid leukocyte activation" as a bridge connected "neutrophil mediated immunity" and other enriched terms. Meanwhile, "neutrophil mediated immunity" also directly connected "cell-cell adhesion" and "myeloid leukocyte migration" by the inter-cluster similarities. The PPI network analysis of the three gene groups established a biological network, consisting of 322 nodes and 584 edges (Figure 4D,E). It contained 78 common genes, most of which (e.g., ANXA1, C3, CCL12, CCL2, CEACAM1, CTSG, CXCR2, ELANE, GATA6, MMP9, NFIB, S100A8, and S100A9) were significantly upregulated. Compared to rL-H5 alone group, 22 genes including AICDA, CCL4, CSF3, CXCL10, CXCL11, IFN- $\gamma$, IL-10, IL-12RB1, SAA3, and T-bet were specifically upregulated in AJSAF+rL-H5 group, but not significantly changed or even downregulated in AJSAF/rL-H5 group. The mRNA expression of CXCL1, CXCL9, IL-17 $\alpha$, IL-1 $\alpha$, and PTGS2 were specifically downregulated in AJSAF/rL-H5 group compared to rL-H5 alone group. IRF8, RELA, and JUN were identified as central hubs of the network. For AJSAF+rL-H5 group, a cluster of upregulated genes including AICDA, CHI314, CXCL9, HK3, IDO1, IL-12 $\beta$, LDHC, LYZ2, MLKL, SLC11A1, SLC5A12, and TNF was connected by IRF8. Both RELA and JUN could upregulate the mRNA expression of CCL2, CXCL10, IL-10, IL-12 $\beta$, IL-6, and TNF. In addition, RELA specifically regulated the mRNA expression of CSF3, HAMP, IDO1, LCN2, RAG1, and T-bet, while JUN specifically upregulated the mRNA expression of CCL12, CCL4, CCL7, CXCL9, and IFN- $\gamma$.

The molecular mechanisms of AJSAF colocalized with antigen or not were compared via integrating the microarray data (Figure 4F). The upregulated S100A8 and S100A9 in both AJSAF+rL-H5 and AJSAF/rL-H5 groups could be recognized by TLR4, leading to activation of NF-KB (RELA) and AP-1 (JUN and FOS) and induction of Th1 response. Th1-related genes (e.g., ANXA1, EBI3, CCL2, CCL7, and CCL12) and Th2-related genes (e.g., RSAD2) were upregulated, and IL-5, a typical gene of Th2 response, was downregulated in both AJSAF+rL-H5 and AJSAF/rL-H5 groups. In AJS+rL-H5 group, Th1 (e.g., T-bet, IFN- $\gamma$, TNF- $\alpha$, IL-12 $\beta$, IL12R $\beta 1$, CCL4, CXCL9, CXCL10, and CXCL11), Th2 (IDO1, EDN1, IL-6, and IL-10), and Th17 (IL-6, IL-12 $\beta$, and IL12R $\beta 1$ ) immune response genes (IRGs) were upregulated, and SMAD7, a negative regulator for Th17 response, was downregulated. In AJSAF/rL-H5 group, several IRGs including GATA3, IL-1 $\alpha$, PTGS2, CD86, IL-17 $\alpha$, CXCL1, and IL-10 were downregulated. 


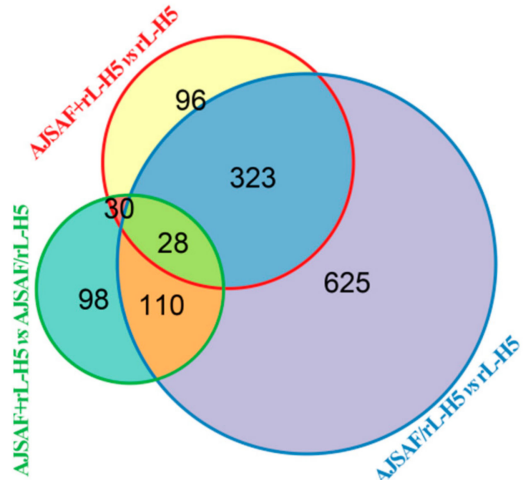

(A)

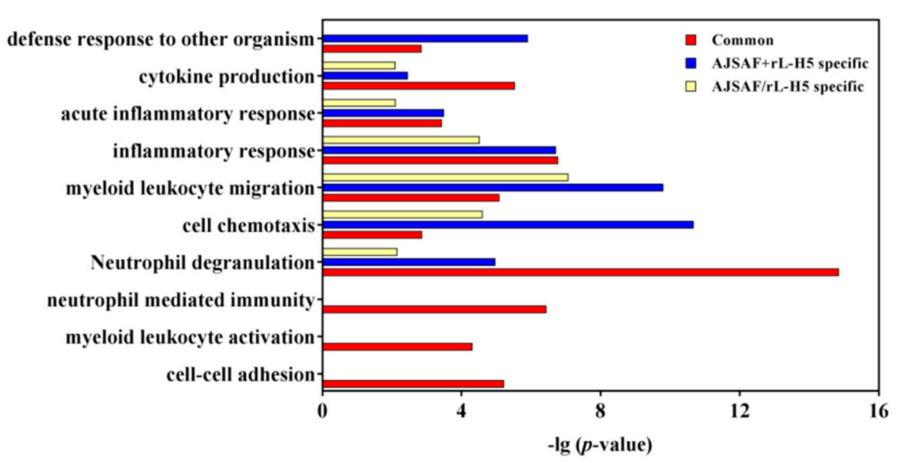

(B)

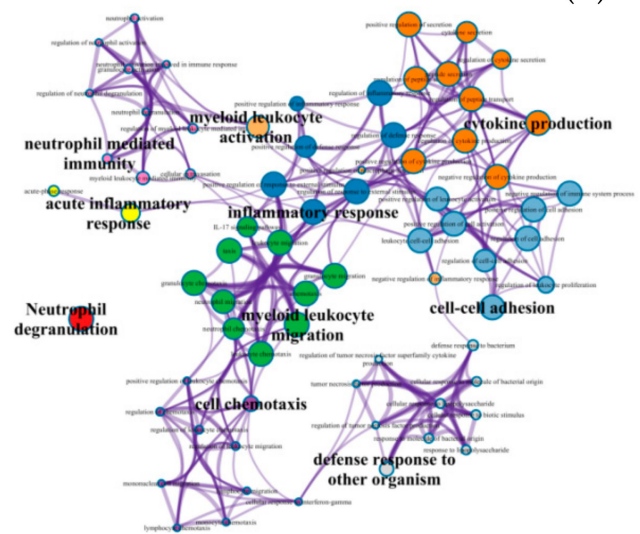

(C)

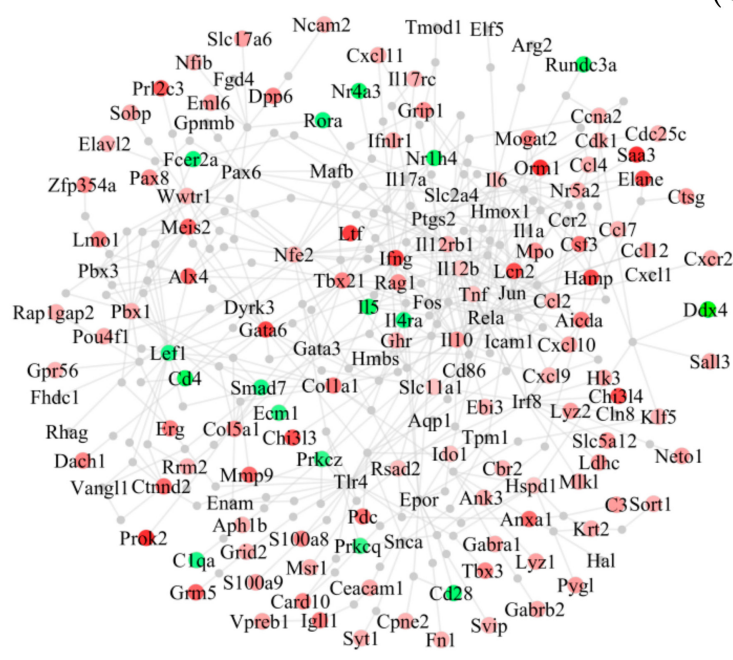

(D)

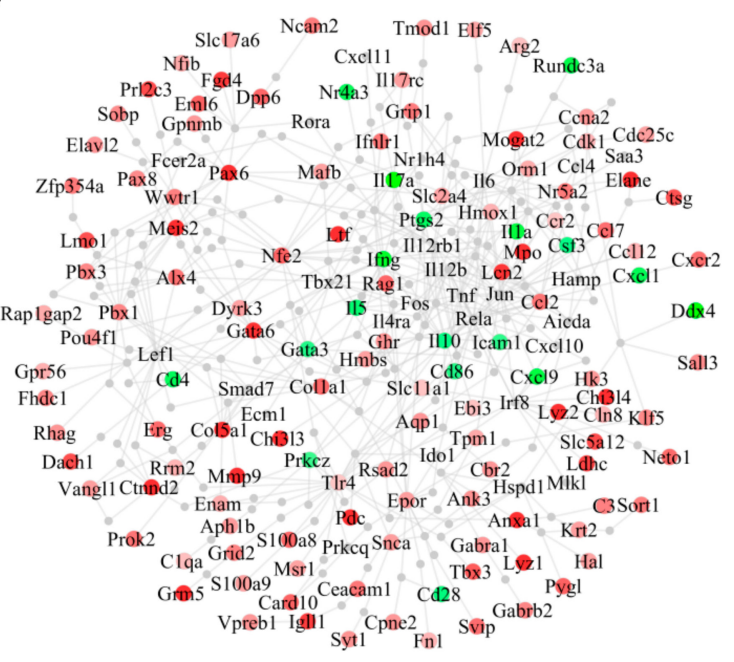

(E)

Figure 4. Cont. 


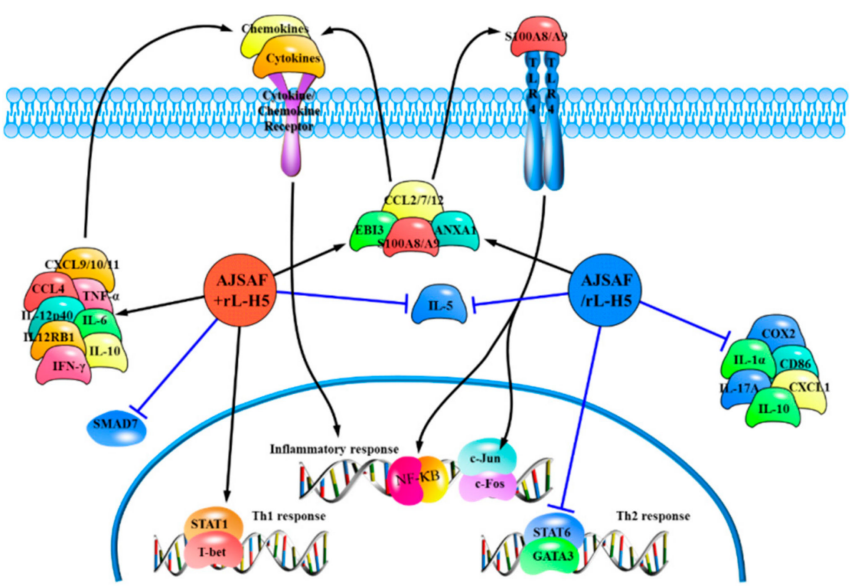

(F)

Figure 4. Function and pathway of differentially expressed genes (DEGs) in H5Ag-stimulated splenocytes from the mice immunized with rL-H5 alone (rL-H5) or in combination with AJSAF at the same leg (AJSAF+rL-H5) or different legs (AJSAF/rL-H5). (A) Venn diagram. (B,C) Enriched function and pathway (B) and network visualization (C) of DEGs. (D,E) Protein-protein interaction (PPI) network of DEGs in AJSAF+rL-H5 (D) and AJSAF/rL-H5 (E) compared to rL-H5 alone with upregulated and downregulated DEGs expressed in red and green, respectively. (F) Proposed mechanisms of adjuvant activity of AJSAF based on the transcriptomic profile.

\subsection{Integrative Analysis of Transcriptomic and Proteomic Profiles}

The proteomic analysis of H5Ag-stimulated splenocytes from the mice immunized with rL-H5 was performed using 2D DIGE-MALDI-TOF-MS (Figure 5A). Among 1637 detected protein spots, 52 differentially expressed protein (DEP) spots were found in AJSAF+rL-H5 group compared with rL-H5 alone group with FC $>1.2$ and $p<0.05$ calculated on the three replicates. Among the DEP spots, a total of 48 spots were identified, including 23 upregulated and 25 downregulated, corresponding to 18 and 23 proteins, respectively (Table 1 ).

To compare the transcriptomic and proteomic profiles, 477 DEGs and 41 DEPs in AJSAF+rL-H5 group compared with rL-H5 alone group were used. It revealed only five in common, including NGP, S100A8, S100A9, ANXA1, and CAMP. The limited consistence of transcriptomic and proteomic data might result from the marked spatial, temporal, and quantitative differences between mRNA and protein expression [27]. On the other hand, it was also restricted by some technical factors such as detection depth difference, screening standard for differential expression, and the limited detection time points. Thus, it highlights the integrated analysis of transcriptomic and proteomic profiles [28]. Beyond single-gene-level analysis, the expression of pathways can be also evaluated directly, which provides a more specific biological context and increasing statistical power [29]. The pathway and function enrichment analysis of DEGs and DEPs was performed, and the comparative results of enriched terms were shown in Figure 5B. Both DEGs and DEPs were significantly enriched in "cell-cell adhesion", "IL-17 signaling pathway", "myeloid leukocyte migration", and "Neutrophil degranulation" $(p<0.001)$. The terms "defense response to other organism", "acute inflammatory response", "myeloid leukocyte activation", and "neutrophil activation" were specifically enriched for DEGs, and "response to interleukin-7" and "actin cytoskeleton organization" for DEPs $(p<0.001)$. The same and different enrichment results between DEGs and DEPs suggested the temporal order and duration of processes induced by AJSAF. As the enrichment network visualization shown in Figure 5C, the terms "response to interleukin-7" and "actin cytoskeleton organization" specifically enriched for DEPs were independent without connections with other enriched terms based on the latest databases. By setting DEPs as primary nodes and DEGs as the second, the PPI network of 226 nodes and 342 edges were established (Figure 5D). It included 10 upregulated (ACTG1, ANXA1, CAPZA2, ECH1, GSN, P4HB, S100A8, S100A9, SERPINB1A, and SRSF1) and 11 downregulated (CORO1A, COTL1, ENO1, EZR, GPX1, 
HMGB1, PARK7, RBM3, RDX, SOD1, and TPI1) DEPs. It revealed that S100A8/A9 was differentially expressed in both gene and protein levels, and connected to TLR4, a critical PPR for Th1 response. Most DEPs indirectly connected with DEGs, but SERPINB1A directly connected with AICDA, CTSG, and ELANE.

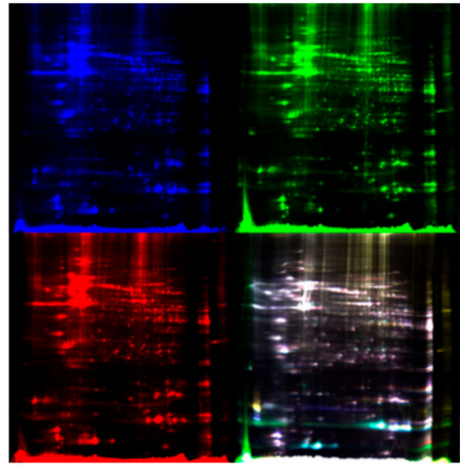

(A)

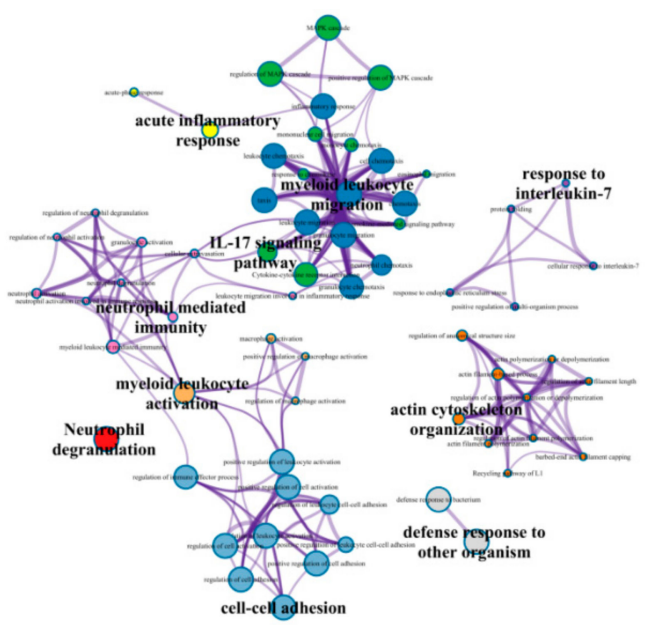

(C)

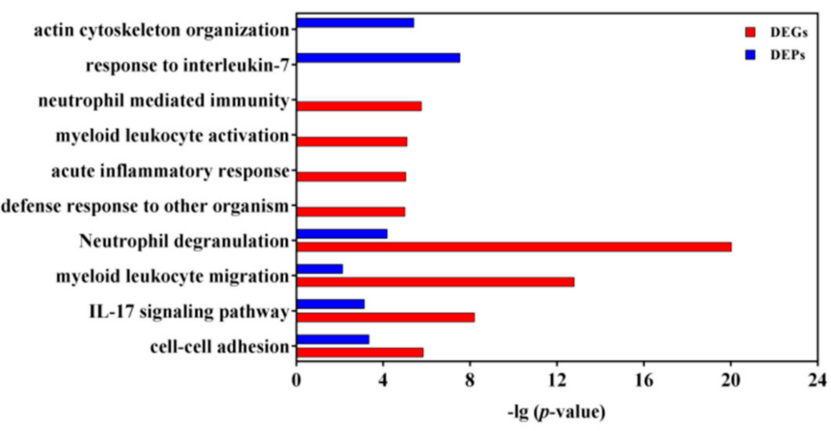

(B)

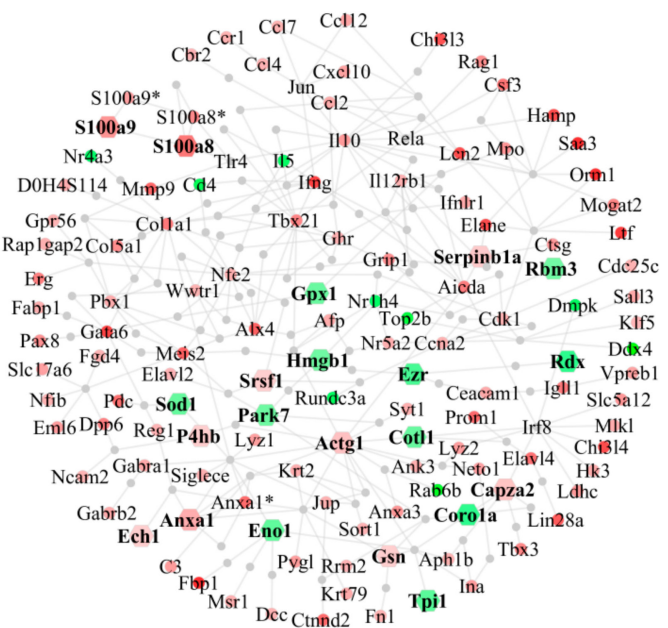

(D)

Figure 5. Function and pathway of differentially expressed proteins (DEPs) in H5Ag-stimulated splenocytes from the mice immunized with rL-H5 alone (rL-H5) or in combination with AJSAF at the same leg (AJSAF+rL-H5). (A) Two-dimensional difference gel electrophoresis (2D DIGE) of proteins. (B,C) Enriched function and pathway $(\mathbf{B})$ and network visualization $(\mathbf{C})$ of DEGs and DEPs. (D) Protein-protein interaction (PPI) network of DEGs (circle) and DEPs (hexagon) with upregulated and downregulated in red and green, respectively. 
Table 1. Differentially expressed protein spots successfully identified by matrix-assisted laser desorption/ionization time-of-flight mass spectrometry (MALDI-TOF-MS).

\begin{tabular}{|c|c|c|c|c|c|c|c|c|c|c|c|}
\hline Spot No. & $p$-Value & Up/Down & Abbr & $\begin{array}{c}\text { Accession } \\
\text { No. }\end{array}$ & ID & MW & PI & $\begin{array}{c}\text { Pep } \\
\text { Count }\end{array}$ & $\begin{array}{c}\text { Protein } \\
\text { Score }\end{array}$ & $\begin{array}{l}\text { Protein/Ion } \\
\text { Credibility }\end{array}$ & $\begin{array}{c}\text { Best Ion } \\
\text { Score }\end{array}$ \\
\hline L21 & 0.0031 & 3.38 & NGP & IPI00127280 & 18054 & 19661.7 & 5.21 & 18 & 731 & $100 / 100$ & 518 \\
\hline L24 & 0.00054 & 3.91 & & & & 10345.1 & 5.43 & 5 & 206 & $100 / 100$ & 154 \\
\hline M23 & 0.0017 & 3.05 & & & & 10345.1 & 5.43 & 5 & 322 & $100 / 100$ & 270 \\
\hline M24 & 0.017 & 3.02 & & & & 13211.3 & 6.64 & 9 & 383 & $100 / 100$ & 311 \\
\hline $\mathrm{N} 2$ & 0.034 & 2.34 & SI00A9 & 1Р100222556 & 20202 & 13211.3 & 6.64 & 10 & 426 & $100 / 100$ & 339 \\
\hline L15 & 0.0032 & 2.31 & ANXA1 & IPI00230395 & 16952 & 38995.1 & 6.97 & 24 & 826 & $100 / 100$ & 606 \\
\hline N8 & 0.00095 & 1.91 & & & & 18173.8 & 6.2 & 7 & 414 & $100 / 100$ & 356 \\
\hline N9 & 0.00075 & 1.76 & GM9234 & ТРI00987580 & 668548 & 18173.8 & 6.2 & 8 & 526 & $100 / 100$ & 456 \\
\hline L23 & 0.0096 & 1.8 & CAMP & IPI00875797 & 12796 & 19797.3 & 8.9 & 3 & 159 & $100 / 100$ & 142 \\
\hline M16 & 0.034 & 1.67 & ACTG1 & IPI01027491 & 11465 & 32941.3 & 5.15 & 6 & 107 & $100 / 100$ & 75 \\
\hline M15 & 0.049 & 1.66 & FTH1 & IPI00230145 & 14319 & 21224.3 & 5.53 & 7 & 236 & $100 / 100$ & 187 \\
\hline M6 & 0.037 & 1.63 & ALDH2 & IPI00111218 & 11669 & 57014.9 & 7.53 & 23 & 849 & $100 / 100$ & 647 \\
\hline L14 & 0.013 & 1.56 & CAPZA2 & IPI00111265 & 12343 & 33117.7 & 5.57 & 11 & 347 & $100 / 100$ & 260 \\
\hline M8 & 0.05 & 1.39 & SERPINB1A & IPI00457659 & 66222 & 42718.8 & 5.85 & 22 & 1,130 & $100 / 100$ & 928 \\
\hline L17 & 0.0012 & 1.38 & PNP & IPI00607023 & 18950 & 32527.2 & 5.78 & 16 & 680 & $100 / 100$ & 513 \\
\hline M10 & 0.0066 & 1.27 & GSN & IPI00759948 & 227753 & 80997.5 & 5.52 & 7 & 113 & 100/100 & 94 \\
\hline N19 & 0.042 & 1.25 & ECH1 & IPI00130804 & 51798 & 36437.3 & 7.6 & 14 & 603 & $100 / 100$ & 493 \\
\hline M5 & 0.02 & 1.24 & P4HB & IPI00133522 & 18453 & 57421.9 & 4.77 & 31 & 905 & $100 / 100$ & 595 \\
\hline N16 & 0.044 & 1.2 & & & & 28927.9 & 6.67 & 15 & 812 & $100 / 100$ & 666 \\
\hline $\mathrm{O} 3$ & 0.022 & 1.25 & PGAM1 & IPI00457898 & 18648 & 28927.9 & 6.67 & 17 & 819 & $100 / 100$ & 643 \\
\hline N14 & 0.0042 & -2.12 & BLVRB & IPI00113996 & 233016 & 22297.4 & 6.49 & 8 & 321 & $100 / 100$ & 255 \\
\hline N13 & 0.0079 & -1.83 & IGK-C & IPI00850020 & 16071 & 24434.9 & 7.05 & 8 & 256 & $100 / 100$ & 192 \\
\hline M4 & 0.03 & -1.55 & CORO1A & IPI00323600 & 12721 & 51641.2 & 6.05 & 15 & 447 & $100 / 100$ & 339 \\
\hline M2 & 0.0017 & -1.54 & RDX & IPI00308324 & 19684 & 68614.4 & 5.91 & 15 & 247 & $100 / 100$ & 183 \\
\hline M20 & 0.024 & -1.48 & SNRPF & IPI00943994 & 69878 & 9775.8 & 4.7 & 4 & 110 & $100 / 100$ & 76 \\
\hline L11 & 0.0027 & -1.45 & EZR & IPI00330862 & 22350 & 69477.7 & 5.83 & 10 & 217 & $100 / 100$ & 186 \\
\hline N12 & 0.019 & -1.38 & GUK1 & IPI00986878 & 14923 & 22018.3 & 6.12 & 5 & 168 & $100 / 100$ & 136 \\
\hline L22 & 0.0036 & -1.38 & RGS10 & IPI00132450 & 67865 & 21194.6 & 6.36 & 15 & 500 & $100 / 100$ & 347 \\
\hline N15 & 0.0065 & -1.36 & TPI1 & IPI00988063 & 21991 & 27037.9 & 6.9 & 15 & 643 & $100 / 100$ & 494 \\
\hline
\end{tabular}


Table 1. Cont.

\begin{tabular}{|c|c|c|c|c|c|c|c|c|c|c|c|}
\hline Spot No. & $p$-Value & Up/Down & Abbr & $\begin{array}{c}\text { Accession } \\
\text { No. }\end{array}$ & ID & MW & PI & $\begin{array}{c}\text { Pep } \\
\text { Count }\end{array}$ & $\begin{array}{c}\text { Protein } \\
\text { Score }\end{array}$ & $\begin{array}{l}\text { Protein/Ion } \\
\text { Credibility }\end{array}$ & $\begin{array}{c}\text { Best Ion } \\
\text { Score }\end{array}$ \\
\hline M7 & 0.0051 & -1.33 & ENO1 & IPI00462072 & 13806 & 47453.3 & 6.37 & 21 & 628 & $100 / 100$ & 431 \\
\hline N6 & 0.0013 & -1.38 & PPIA & IPIO0554989 & 268373 & 18473.1 & 7.74 & 9 & 336 & $100 / 100$ & 255 \\
\hline N18 & 0.0099 & -1.3 & EIF4H & IPI00124742 & 22384 & 27381.4 & 6.67 & 14 & 350 & $100 / 100$ & 240 \\
\hline N3 & 0.0012 & -1.28 & & & & 16103.9 & 6.02 & 13 & 645 & $100 / 100$ & 500 \\
\hline N4 & 0.028 & -1.3 & SOD1 & IPI00130589 & 20655 & 16103.9 & 6.02 & 8 & 164 & $100 / 100$ & 93 \\
\hline N11 & 0.0063 & -1.28 & GPX1 & IPI00319652 & 14775 & 22553.4 & 6.74 & 7 & 457 & $100 / 100$ & 404 \\
\hline M12 & 0.013 & -1.28 & PRDX2 & IPI00117910 & 21672 & 21936.1 & 5.2 & 9 & 593 & $100 / 100$ & 515 \\
\hline M14 & 0.029 & -1.26 & PARK7 & IPI00117264 & 57320 & 20236.5 & 6.32 & 9 & 334 & $100 / 100$ & 261 \\
\hline M3 & 0.011 & -1.25 & PDIA3 & IPI00230108 & 14827 & 57098.9 & 5.88 & 28 & 692 & $100 / 100$ & 426 \\
\hline N17 & 0.052 & -1.24 & HMGB1 & IPI00420261 & 15289 & 25049.2 & 5.62 & 11 & 220 & $100 / 100$ & 144 \\
\hline M19 & 0.016 & -1.24 & SH3BGRL3 & IPI00127358 & 73723 & 10527.3 & 5.02 & 6 & 274 & $100 / 100$ & 214 \\
\hline M13 & 0.00047 & -1.23 & CMPK1 & IPI00331146 & 66588 & 26040.4 & 8.13 & 14 & 511 & $100 / 100$ & 394 \\
\hline N5 & 0.032 & -1.22 & RBM3 & IPI00130883 & 19652 & 16594.7 & 6.84 & 6 & 158 & $100 / 100$ & 114 \\
\hline N10 & 0.021 & -1.2 & ARPC5L & IPI00111117 & 74192 & 17026.8 & 6.32 & 7 & 384 & $100 / 100$ & 326 \\
\hline
\end{tabular}




\section{Discussion}

AJSAF is an ideal adjuvant candidate that induces antigen-specific of both cellular and humoral immune responses with mixed Th1/Th2 responses [14,15]. AJSAF has been recently reported to activate RAW264.7 cells via $\mathrm{Ca}^{2+}$-ERK1/2-CREB pathways [30]. However, its in vivo mechanisms of adjuvant activity have not been well clarified yet. Moreover, the mechanisms of Th1 or Th2 selectivity of adjuvants remain inconclusive, resulting in a shortage of guidelines for designing selective adjuvants. In this study, the mechanisms of action of AJSAF were explored by comparing adaptive immune response in mice immunized with $\mathrm{rL}-\mathrm{H} 5$ and AJSAF at the same leg or different legs. It revealed that AJSAF-mediated antibody response was dependent on spatial colocalization of AJSAF and antigen.

AJSAF+rL-H5 specifically upregulated several typical Th1 IRGs including T-bet, IFN- $\gamma$, TNF- $\alpha$, IL-12 $\beta$, and IL-12R $\beta 1$ (Figure 4F). Th1 response was also characterized by the common genes (e.g., S100A8, S100A9, ANXA1, and EBI3) and functions (e.g., "inflammatory response" and "neutrophil mediated immunity"). The upregulated mRNA expression of S100A8, S100A9, and ANXA1 in AJSAF+rL-H5 group was also verified by proteomic data. S100A8 and S100A9 are calcium- and zinc-binding proteins and involve in the regulation of inflammatory processes and immune response. During inflammation, S100A8 and S100A9 regulate the inflammatory response characterized by leukocyte recruitment and cytokine secretion through activating RAGE and TLR4 [31]. S100A8 and S100A9 could induce DC maturation [32] and contribute to the development of autoreactive CD8 ${ }^{+}$ effectors through TLR4 [33]. Recently, S100A8 and S100A9 have been defined as alarmins in driving adaptive immune responses. Alarmins are a subset of endogenous DAMPs that interact with PRRs such as TLRs as cytokine-like mediators that participate in host defense and are usually not dangerous [34]. Alarmins are distinct from other endogenous DAMPs in their efficacy as endogenous immunoenhancing adjuvants $[35,36]$. S100A8 and S100A9 were reported to be Th1-polarizing alarmins to shape the types of adaptive immune response [34]. Therefore, AJSAF-induced predominant elevation of S100A8 and S100A9 was proposed to be required for its adjuvanticity. Another Th1-polarizing alarmin, high-mobility group box 1 protein (HMGB1), has also been demonstrated to exhibit adjuvant activity [37]. However, HMGB1 was significantly downregulated in AJSAF+rL-H5 group, thus suggesting its distinct role. More studies are warranted to clarify the role of alarmins in AJSAF-mediated immune responses.

The "inflammatory response" is another common property, independent of colocalization of AJSAF and rL-H5. The role of inflammation in adjuvant activity remains inconclusive. However, it is undoubted and unarguable that the overly aggressive or prolonged inflammation affects the safety of adjuvants. Annexin A1 (ANXA1) is an anti-inflammatory mediator with pro-resolving properties. ANXA1 has been shown to exert various anti-inflammatory actions: (i) Inhibited neutrophil recruitment, (ii) induced neutrophil apoptosis, (iii) promoted monocyte recruitment, (iv) activated the clearance of apoptotic leukocytes by macrophages, and (v) yielded macrophage reprogramming from a pro-inflammatory to a pro-resolving phenotype [38]. Evidence indicated that the externalization of ANXA1 and then its interaction with the formyl peptide receptor type 2/lipoxin A4 receptor (FPR2/ALX) were required for its anti-inflammatory effects [39-41]. The microarray data revealed that AJSAF significantly upregulated the mRNA expression of ANXA1 and FPR2 in mice immunized with rL-H5 suggesting the involvement of ANXA1-FPR2/ALX signaling in the anti-inflammatory effects of AJSAF. The upregulation of neutrophil elastases (ELANE, CTSG, and PRTN3) and their fast-acting inhibitor SERPINB1A indicated another anti-inflammatory mechanism of AJSAF [42]. Accompanied by the initiation of acute inflammation, AJSAF induced effective anti-inflammatory response to prevent the excessive and prolonged inflammation, reducing its side effects.

Unlike Th1 response, there were limited numbers of classical Th2 genes upregulated in AJSAF+rL-H5 group, and it failed to discover the precise signaling events or crucial factors to interpret the mechanisms of action of AJSAF-mediated Th2 responses. IL-10 helps to polarize Th2 immune response by preferentially suppressing the production of IL-12 and IFN- $\gamma$ from Th1 cells [43]. However, Khan et al. [44] reported that the endogenous IL-10 is not a switch factor for IgG1. Thus, except IL-10, other mediators might contribute to Th2-polarized antibodies induced by AJSAF. Accumulating 
evidence suggests that most adjuvants trigger early innate immune responses to induce robust and long-lasting adaptive immune responses [45]. The observation that AJSAF-mediated Th2 response was dependent on the colocalization of AJSAF with antigen suggested a causative role of the local innate immune response triggered by AJSAF. In our previous study, adjuvant-active fraction from $A$. julibrissin saponin induced the production of Th2 cytokines (IL-13 and IL-9) at the injection site [14]. Whether the local innate immune response triggered by AJSAF regulates subsequent Th2 responses is being elucidated.

In addition, "actin cytoskeleton organization" might mediate Th1 and Th2 immune responses induced by AJSAF. Actin, a major component of the cytoskeleton, is a dynamic polymer. Its monomer is globular (G-actin, encoded by ACTG1) and forms various shaped filaments (F-actin) when polymerized. The dynamic nature of actin polymerization and depolymerization is central to functions of actin network, which is regulated by numerous actin binding proteins (ABPs). Recently, the importance of cytoskeletal function in immunity has been well-recognized. Beyond enabling cell migration and adhesion, the actin network is essential for many facets of innate and adaptive immunity, including phagocytosis, leukocyte activation, and immune synapse formation [46]. Gelsolin (GSN), one of the most abundant ABPs, regulates actin by severing, capping, nucleating actin filaments, and sequestering monomers [47]. It was reported that GSN participated in immunological processes such as phagocytosis [48], macrophage recruitment, and motility [49], as well as neutrophil regulation and adhesion [50]. Capping actin protein of muscle Z-line subunit alpha 2 (CAPZ) binds to the fast-growing barbed ends of actin filaments thereby blocking the exchange of subunits at these ends. Coactosin-like protein 1 (COTL1), a member of the actin-depolymerizing factor (ADF)/cofilin family, was shown to bind F-actin, but not G-actin [51]. COTL1 competes with cofilin for binding to F-actin, and then attenuates cofilin-mediated F-actin depolymerization to promote lamellipodial protrusion [52]. Coronin 1A (CORO1A) is a member of the coronin family that function as important regulators of the actin cytoskeleton. It regulated the innate and adaptive immune responses in an actin-dependent manner [53], such as enhancing neutrophil phagocytosis [54], and regulating chemokine-mediated T-cell migration [55]. Ezrin (EZR), radixin (RDX) and moesin (MSN) belong to the ezrin-radixin-mesin (ERM) family of membrane-actin cytoskeleton crosslinkers and participate in a variety of cellular processes. These ABPs control the shape, cytokinesis, adhesion, and activation of T cell [56] and participate in immune synapse formation, an essential process for APC-T cell interaction [57]. It was reported that the size of $\mathrm{B}$ cell receptor (BCR) microclusters, and magnitude of BCR signaling and antigen-specific antibody production are increased in the absence of EZR [58] and that the conditional deletion of EZR in B cells increases IL-10 production induced by TLR4 ligation [59]. Although the protein level changes of G-actin and these ABPs were observed in our data, their functions in mediating the adaptive immune response remain to be elucidated.

\section{Conclusions}

The molecular mechanisms of action of AJSAF were comprehensively analyzed based on transcriptomic and proteomic profiles from the single gene level to the pathway, function, and network levels (Figure 6). In addition to the activation of S100A8/A9-TLR4-NF-kB/AP-1 pathway and production of Th1/Th2 cytokines, AJSAF was proposed to regulate: (i) The actin cytoskeleton, characterized by upregulation of ACTG1, CAPZ, and GSN, as well as downregulation of CORO1A, COTL1, EZR, and RDX; (ii) the leukocyte migration through affecting actin cytoskeleton and inducing chemokines (e.g., CCL2, CCL4, and CCL7); (iii) the anti-inflammatory response, including ANXA1-mediated anti-inflammatory effects via FPR2/ALX and SERPINB1A-mediated suppression of neutrophil elastases (ELANE and CTSG). 


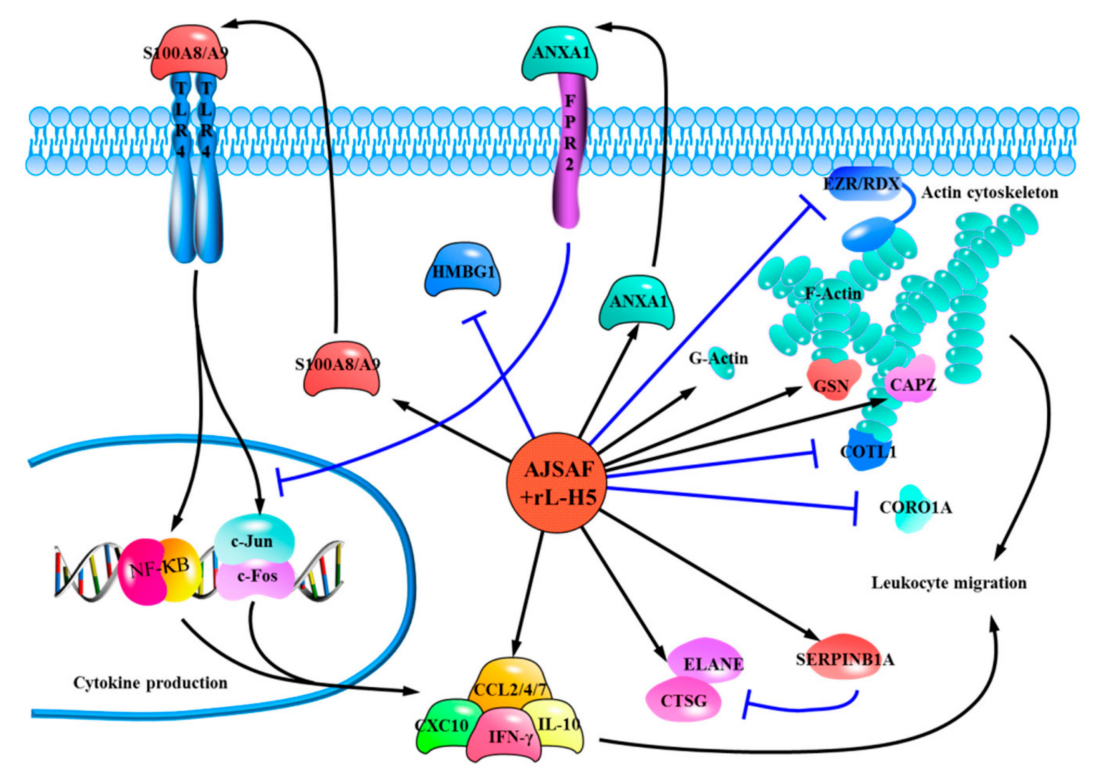

Figure 6. Proposed mechanisms of the adaptive immune response in mice induced by AJSAF based on the integrative analysis of transcriptomic and proteomic profiles.

In this study, the mechanisms of action of Th1/Th2 responses induced by AJSAF were demonstrated based on the transcriptomic and proteomic profiles. The neutrophil response and its derived alarmin S100A8 and S100A9 might involve in the Th1 response. The dual nature of neutrophils is consistent with the benefit/risk profile of saponin-based adjuvants. Meanwhile, AJSAF might induce the adaptive immune responses by improving a local innate immune microenvironment. Our findings also highlight the important role of various alarmins in adjuvant studies due to their adjuvant efficacy, identified receptors, and downstream signal transducers. The insights obtained from this study further advance our understanding of the mechanisms of action of saponin-based adjuvants.

Supplementary Materials: The following are available online at http://www.mdpi.com/2076-393X/8/1/48/s1, Table S1. Primers used for qRT-PCR; Figure S1. Heatmap of verified genes in microarray analysis; Figure S2. Heatmap of gene sets on "adaptive immune response (GO: 0002250)" using gene set enrichment analysis (GSEA).

Author Contributions: J.D. conducted all experiments, acquired the data, analyzed the results, and drafted the manuscript; J.J. and J.W. performed transcriptomic and proteomic information experiments; H.S. designed the study, performed all experiments, and revised the manuscript. All authors have read and agreed to the published version of the manuscript.

Funding: This work was supported by Grant-in-Aid from the National Natural Science Foundation of China (Nos. 31472229, 31772783, and 31972726), the Zhejiang Provincial Natural Science Foundation of China (No. LZ13C180001), the National Key R\&D Program of China (2017YFD0501505), and the Dabeinong Funds for Discipline Development and Talent Training in Zhejiang University.

Conflicts of Interest: The authors declare no conflict of interest.

\section{References}

1. Oyston, P.; Robinso, K. The current challenges for vaccine development. J. Med. Microbiol. 2012, 61, 889-894. [CrossRef] [PubMed]

2. Mosmann, T.R.; Sad, S. The expanding universe of T-cells subsets: Th1, Th2 and more. Immunol. Today 1996, 17, 138-146. [CrossRef]

3. McKee, A.S.; Munks, M.W.; Marrack, P. How do adjuvants work? Important considerations for new generation adjuvants. Immunity 2007, 27, 687-690. [CrossRef] [PubMed]

4. McKee, A.S.; Marrack, P. Old and new adjuvants. Curr. Opin. Immunol. 2017, 47, 44-51. [CrossRef]

5. Reed, S.G.; Bertholet, S.; Coler, R.N.; Friede, M. New horizons in adjuvants for vaccine development. Trends Immunol. 2009, 30, 23-32. [CrossRef] 
6. Kool, M.; Pétrilli, V.; De Smedt, T.; Rolaz, A.; Hammad, H.; van Nimwegen, M.; Bergen, I.M.; Castillo, R.; Lambrecht, B.N.; Tschopp, J. Cutting edge: alum adjuvant stimulates inflammatory dendritic cells through activation of the NALP3 inflammasome. J. Immunol. 2008, 181, 3755-3759. [CrossRef]

7. Eisenbarth, S.C.; Colegio, O.R.; O'Connor, W.; Sutterwala, F.S.; Flavell, R.A. Crucial role for the Nalp3 inflammasome in the immunostimulatory properties of aluminium adjuvants. Nature 2008, 453, 1122-1126. [CrossRef]

8. Li, H.; Willingham, S.B.; Ting, J.P.Y.; Re, F. Cutting edge: inflammasome activation by alum and alum's adjuvant effect are mediated by NLRP3. J. Immunol. 2008, 181, 17-21. [CrossRef]

9. Kool, M.; Soullié, T.; van Nimwegen, M.; Willart, M.A.; Muskens, F.; Jung, S.; Hoogsteden, H.C.; Hammad, H.; Lambrecht, B.N. Alum adjuvant boosts adaptive immunity by inducing uric acid and activating inflammatory dendritic cells. J. Exp. Med. 2008, 205, 869-882. [CrossRef]

10. Marichal, T.; Ohata, K.; Bedoret, D.; Mesnil, C.; Sabatel, C.; Kobiyama, K.; Lekeux, P.; Coban, C.; Akira, S.; Ishii, K.J.; et al. DNA released from dying host cells mediates aluminum adjuvant activity. Nat. Med. 2011, 17, 996-1002. [CrossRef]

11. McKee, A.S.; Burchill, M.A.; Munks, M.W.; Jin, L.; Kappler, J.W.; Friedman, R.S.; Jacobelli, J.; Marrack, P. Host DNA released in response to aluminum adjuvant enhances MHC class II-mediated antigen presentation and prolongs CD4 T-cell interactions with dendritic cells. Proc. Natl. Acad. Sci. USA 2013, 110, E1122-E1131. [CrossRef] [PubMed]

12. Franchi, L.; Núñez, G. The Nlrp3 inflammasome is critical for aluminium hydroxide-mediated IL-1 $\beta$ secretion but dispensable for adjuvant activity. Eur. J. Immunol. 2008, 38, 2085-2089. [CrossRef] [PubMed]

13. McKee, A.S.; Munks, M.W.; MacLeod, M.K.; Fleenor, C.J.; Van Rooijen, N.; Kappler, J.W.; Marrack, P. Alum induces innate immune responses through macrophage and mast cell sensors, but these sensors are not required for alum to act as an adjuvant for specific immunity. J. Immunol. 2009, 183, 4403-4414. [CrossRef] [PubMed]

14. Sun, H.X.; He, S.W.; Shi, M.H. Adjuvant-active fraction from Albizia julibrissin saponins improves immune responses by inducing cytokine and chemokine at the site of injection. Int. Immunopharmacol. 2014, 22, 346-355. [CrossRef] [PubMed]

15. Zhu, B.N.; He, T.Y.; Gao, X.Y.; Shi, M.H.; Sun, H.X. Evaluation and characteristics of immunological adjuvant activity of purified fraction of Albizia julibrissin saponins. Immunol. Invest. 2019, 48, 283-302. [CrossRef] [PubMed]

16. Morel, S.; Didierlaurent, A.; Bourguignon, P.; Delhaye, S.; Baras, B.; Jacob, V.; Planty, C.; Elouahabi, A.; Harvengt, P.; Carlsen, H.; et al. Adjuvant System AS03 containing $\alpha$-tocopherol modulates innate immune response and leads to improved adaptive immunity. Vaccine 2011, 29, 2461-2473. [CrossRef]

17. He, Y.F.; Liu, Z.Y.; Ye, Y.P.; Sun, H.X. Rapid annotation and structural characterization of saponins in the active fraction of Albizia julibrissin by HPLC coupled with quadrupole time-of-flight mass spectrometry based on accurate mass database. J. Sep. Sci. 2019, 42, 2922-2941. [CrossRef]

18. Xie, Y.; Sun, H.X.; Li, D. Platycodin D improves the immunogenicity of Newcastle disease virus-based recombinant avian influenza vaccine in mice. Chem. Biodiv. 2010, 7, 677-689. [CrossRef]

19. Du, J.; Chen, X.F.; Wang, C.Y.; Sun, H.X. Pathway analysis of global gene expression change in dendritic cells induced by the polysaccharide from the roots of Actinidia eriantha. J. Ethnopharmacol. 2018, 214, 141-152. [CrossRef]

20. Subramanian, A.; Tamayo, P.; Mootha, V.K.; Mukherjee, S.; Ebert, B.L.; Gillette, M.A.; Paulovich, A.; Pomeroy, S.L.; Golub, T.R.; Lander, E.S.; et al. Gene set enrichment analysis: a knowledge-based approach for interpreting genome-wide expression profiles. Proc. Natl. Acad. Sci. USA 2005, 102, 15545-15550. [CrossRef]

21. Zhou, Y.; Zhou, B.; Pache, L.; Chang, M.; Khodabakhshi, A.H.; Tanaseichuk, O.; Benner, C.; Chanda, S.K. Metascape provides a biologist-oriented resource for the analysis of systems-level datasets. Nat. Commun. 2019, 10, 1523. [CrossRef] [PubMed]

22. Breuer, K.; Foroushani, A.K.; Laird, M.R.; Chen, C.; Sribnaia, A.; Lo, R.; Winsor, G.L.; Hancock, R.E.; Brinkman, F.S.; Lynn, D.J. InnateDB: systems biology of innate immunity and beyond-recent updates and continuing curation. Nucleic Acids Res. 2012, 41, D1228-D1233. [CrossRef] [PubMed]

23. Shannon, P.; Markiel, A.; Ozier, O.; Baliga, N.S.; Wang, J.T.; Ramage, D.; Amin, N.; Schwikowski, B.; Ideker, T. Cytoscape: A software environment for integrated models of biomolecular interaction networks. Genome Res. 2003, 13, 2498-2504. [CrossRef] [PubMed] 
24. Szabo, S.J.; Kim, S.T.; Costa, G.L.; Zhang, X.; Fathman, C.G.; Glimcher, L.H. A novel transcription factor, T-bet, directs Th1 lineage commitment. Cell 2000, 100, 655-669. [CrossRef]

25. Jang, E.J.; Park, H.R.; Hong, J.H.; Hwang, E.S. Lysine 313 of T-box is crucial for modulation of protein stability, DNA binding, and threonine phosphorylation of T-bet. J. Immunol. 2013, 190, 5764-5770. [CrossRef]

26. Lazarevic, V.; Chen, X.; Shim, J.H.; Hwang, E.S.; Jang, E.; Bolm, A.N.; Oukka, M.; Kuchroo, V.K.; Glimcher, L.H. T-bet represses $\mathrm{T}(\mathrm{H}) 17$ differentiation by preventing Runx1-mediated activation of the gene encoding ROR $\gamma \mathrm{t}$. Nat. Immunol. 2011, 12, 96-104. [CrossRef]

27. De Sousa Abreu, R.; Penalva, L.O.; Marcotte, E.M.; Vogel, C. Global signatures of protein and mRNA expression levels. Mol. Biosyst. 2009, 5, 1512-1526. [CrossRef]

28. Seyfried, N.T.; Dammer, E.B.; Swarup, V.; Nandakumar, D.; Duong, D.M.; Yin, L.; Deng, Q.; Nguyen, T.; Hales, C.M.; Wingo, T.; et al. A multi-network approach identifies protein-specific co-expression in asymptomatic and symptomatic Alzheimer's disease. Cell Syst. 2017, 4, 60-72. [CrossRef]

29. Li, S.; Rouphael, N.; Duraisingham, S.; Romero-Steiner, S.; Presnell, S.; Davis, C.; Schmidt, D.S.; Johnson, S.E.; Milton, A.; Rajam, G.; et al. Molecular signatures of antibody responses derived from a systems biology study of five human vaccines. Nat. Immunol. 2014, 15, 195-204. [CrossRef]

30. Wang, C.Y.; Du, J.; Chen, X.F.; Zhu, Y.L.; Sun, H.X. Activation of RAW264. 7 macrophages by active fraction of Albizia julibrissin saponin via $\mathrm{Ca}^{2+}$-ERK1/2-CREB-lncRNA pathways. Int. Immunopharmacol. 2019, 77, 105955. [CrossRef]

31. Wang, S.; Song, R.; Wang, Z.; Jing, Z.; Wang, S.; Ma, J. S100A8/A9 in Inflammation. Front. Immunol. 2018, 9, 1298. [CrossRef] [PubMed]

32. Petersen, B.; Wolf, M.; Austermann, J.; van Lent, P.; Foell, D.; Ahlmann, M.; Kupas, V.; Loser, K.; Sorg, C.; Roth, J.; et al. The alarmin Mrp8/14 as regulator of the adaptive immune response during allergic contact dermatitis. EMBO J. 2013, 32, 100-111. [CrossRef] [PubMed]

33. Loser, K.; Vogl, T.; Voskort, M.; Lueken, A.; Kupas, V.; Nacken, W.; Klenner, L.; Kuhn, A.; Foell, D.; Sorokin, L.; et al. The Toll-like receptor 4 ligands Mrp8 and Mrp14 are crucial in the development of autoreactive CD8 ${ }^{+} \mathrm{T}$ cells. Nat. Med. 2010, 16, 713-717. [CrossRef] [PubMed]

34. Yang, D.; Han, Z.; Oppenheim, J.J. Alarmins and immunity. Immunol. Rev. 2017, 280, 41-56. [CrossRef]

35. Oppenheim, J.J.; Yang, D. Alarmins: Chemotactic activators of immune responses. Curr. Opin. Immunol. 2005, 17, 359-365. [CrossRef]

36. Wang, Y.; Rahman, D.; Lehner, T. A comparative study of stress-mediated immunological functions with the adjuvanticity of alum. J. Biol. Chem. 2012, 287, 17152-17160. [CrossRef]

37. Rovere-Querini, P.; Capobianco, A.; Scaffidi, P.; Valentinis, B.; Catalanotti, F.; Giazzon, M.; Dumitriu, I.E.; Müller, S.; Iannacone, M.; Traversari, C.; et al. HMGB1 is an endogenous immune adjuvant released by necrotic cells. EMBO Rep. 2004, 5, 825-830. [CrossRef]

38. Sugimoto, M.A.; Vago, J.P.; Teixeira, M.M.; Sousa, L.P. Annexin A1 and the resolution of inflammation: Modulation of neutrophil recruitment, apoptosis, and clearance. J. Immunol. Res. 2016, 2016, 8239258. [CrossRef]

39. Gavins, F.N.; Yona, S.; Kamal, A.M.; Flower, R.J.; Perretti, M. Leukocyte antiadhesive actions of annexin 1: ALXR-and FPR-related anti-inflammatory mechanisms. Blood 2003, 101, 4140-4147. [CrossRef]

40. Li, Y.; Cai, L.; Wang, H.; Wu, P.; Gu, W.; Chen, Y.; Hao, H.; Tang, K.; Yi, P.; Liu, M.; et al. Pleiotropic regulation of macrophage polarization and tumorigenesis by formyl peptide receptor-2. Oncogene 2011, 30, 3887-3899. [CrossRef]

41. Cooray, S.N.; Gobbetti, T.; Montero-Melendez, T.; McArthur, S.; Thompson, D.; Clark, A.J.; Flower, R.J.; Perretti, M. Ligand-specific conformational change of the G-protein-coupled receptor ALX/FPR2 determines proresolving functional responses. Proc. Natl. Acad. Sci. USA 2013, 110, 18232-18237. [CrossRef] [PubMed]

42. Cooley, J.; Takayama, T.K.; Shapiro, S.D.; Schechter, N.M.; Remold-O'Donnell, E. The serpin MNEI inhibits elastase-like and chymotrypsin-like serine proteases through efficient reactions at two active sites. Biochemistry 2001, 40, 15762-15770. [CrossRef] [PubMed]

43. Fiorentino, D.F.; Zlotnik, A.; Vieira, P.; Mosmann, T.R.; Howard, M.; Moore, K.W.; O'Garra, A. IL-10 acts on the antigen-presenting cell to inhibit cytokine production by Th1 cells. J. Immunol. 1991, 146, 3444-3451. [PubMed] 
44. Khan, A.Q.; Shen, Y.; Wu, Z.Q.; Wynn, T.A.; Snapper, C.M. Endogenous pro-and anti-inflammatory cytokines differentially regulate an in vivo humoral response to Streptococcus pneumoniae. Infect. Immun. 2002, 70, 749-761. [CrossRef] [PubMed]

45. Coffman, R.L.; Sher, A.; Seder, R.A. Vaccine adjuvants: putting innate immunity to work. Immunity 2010, 33, 492-503. [CrossRef] [PubMed]

46. Burns, S.O.; Zarafov, A.; Thrasher, A.J. Primary immunodeficiencies due to abnormalities of the actin cytoskeleton. Curr. Opin. Hematol. 2017, 24, 16-22. [CrossRef]

47. Feldt, J.; Schicht, M.; Garreis, F.; Welss, J.; Schneider, U.W.; Paulsen, F. Structure, regulation and related diseases of the actin-binding protein gelsolin. Expert Rev. Mol. Med. 2019, 20, e7. [CrossRef]

48. Arora, P.D.; Glogauer, M.; Kapus, A.; Kwiatkowski, D.J.; McCulloch, C.A. Gelsolin mediates collagen phagocytosis through a rac-dependent step. Mol. Biol. Cell 2004, 15, 588-599. [CrossRef]

49. Gonçalves, A.F.; Dias, N.G.; Moransard, M.; Correia, R.; Pereira, J.A.; Witke, W.; Suter, U.; Relvas, J.B. Gelsolin is required for macrophage recruitment during remyelination of the peripheral nervous system. Glia 2010, 58, 706-715.

50. Langereis, J.D. Neutrophil integrin affinity regulation in adhesion, migration, and bacterial clearance. Cell Adh. Migr. 2013, 7, 486-491. [CrossRef]

51. Provost, P.; Doucet, J.; Stock, A.; Gerisch, G.; Samuelsson, B.; Rådmark, O. Coactosin-like protein, a human F-actin-binding protein: critical role of lysine-75. Biochem. J. 2001, 359, 255-263. [CrossRef] [PubMed]

52. Kim, J.; Shapiro, M.J.; Bamidele, A.O.; Gurel, P.; Thapa, P.; Higgs, H.N.; Hedin, K.E.; Shapiro, V.S.; Billadeau, D.D. Coactosin-like 1 antagonizes cofilin to promote lamellipodial protrusion at the immune synapse. PLoS ONE 2014, 9, e85090. [CrossRef] [PubMed]

53. Chan, K.T.; Creed, S.J.; Bear, J.E. Unraveling the enigma: progress towards understanding the coronin family of actin regulators. Trends Cell Biol. 2011, 21, 481-488. [CrossRef] [PubMed]

54. Yokoyama, K.; Kaji, H.; He, J.; Tanaka, C.; Hazama, R.; Kamigaki, T.; Ku, Y.; Tohyama, K.; Tohyama, Y. Rab27a negatively regulates phagocytosis by prolongation of the actin-coating stage around phagosomes. J. Biol. Chem. 2011, 286, 5375-5382. [CrossRef]

55. Föger, N.; Rangell, L.; Danilenko, D.M.; Chan, A.C. Requirement for coronin 1 in T lymphocyte trafficking and cellular homeostasis. Science 2006, 313, 839-842. [CrossRef]

56. Li, Y.; Harada, T.; Juang, Y.T.; Kyttaris, V.C.; Wang, Y.; Zidanic, M.; Tung, K.; Tsokos, G.C. Phosphorylated ERM is responsible for increased $\mathrm{T}$ cell polarization, adhesion, and migration in patients with systemic lupus erythematosus. J. Immunol. 2007, 178, 1938-1947. [CrossRef]

57. Cullinan, P.; Sperling, A.I.; Burkhardt, J.K. The distal pole complex: A novel membrane domain distal to the immunological synapse. Immunol. Rev. 2002, 189, 111-122. [CrossRef]

58. Pore, D.; Parameswaran, N.; Matsui, K.; Stone, M.B.; Saotome, I.; McClatchey, A.I.; Veatch, S.L.; Gupta, N. Ezrin tunes the magnitude of humoral immunity. J. Immunol. 2013, 191, 4048-4058. [CrossRef]

59. Pore, D.; Matsui, K.; Parameswaran, N.; Gupta, N. Cutting edge: ezrin regulates inflammation by limiting B cell IL-10 production. J. Immunol. 2016, 196, 558-562. [CrossRef]

(C) 2020 by the authors. Licensee MDPI, Basel, Switzerland. This article is an open access article distributed under the terms and conditions of the Creative Commons Attribution (CC BY) license (http://creativecommons.org/licenses/by/4.0/). 